Proceedings of the Edinburgh Mathematical Society (2006) 49, 331-359 (C)

DOI:10.1017/S0013091505000386 Printed in the United Kingdom

\title{
ENTROPY NUMBERS OF EMBEDDINGS OF WEIGHTED BESOV SPACES. II
}

\author{
THOMAS KÜHN ${ }^{1}$, HANS-GERD LEOPOLD ${ }^{2}$, \\ WINFRIED SICKEL ${ }^{2}$ AND LESZEK SKRZYPCZAK ${ }^{3}$ \\ ${ }^{1}$ Mathematisches Institut, Universität Leipzig, Augustusplatz 10/11, \\ 04109 Leipzig, Germany (kuehn@math.uni-leipzig.de) \\ ${ }^{2}$ Mathematisches Institut, Friedrich-Schiller-Universität Jena, Ernst-Abbe-Platz 1-2, \\ 07743 Jena, Germany (leopold@minet.uni-jena.de; sickel@minet.uni-jena.de) \\ ${ }^{3}$ Faculty of Mathematics and Computer Science, Adam Mickiewicz University, \\ Ul. Umultowska 87, Pl-61-614 Poznan, Poland (lskrzyp@amu.edu.pl)
}

(Received 23 March 2005)

\begin{abstract}
We investigate the asymptotic behaviour of the entropy numbers of the compact embed$\operatorname{ding} B_{p_{1}, q_{1}}^{s_{1}}\left(\mathbb{R}^{d}, w_{1}\right) \hookrightarrow B_{p_{2}, q_{2}}^{s_{2}}\left(\mathbb{R}^{d}, w_{2}\right)$. Here $B_{p, q}^{s}\left(\mathbb{R}^{d}, w\right)$ denotes a weighted Besov space. We present a general approach which allows us to work with a large class of weights.
\end{abstract}

Keywords: smooth weights; indices; weighted Besov spaces; compact embeddings; entropy numbers

2000 Mathematics subject classification: Primary 41A46; 46E35

\section{Introduction}

Nowadays the entropy numbers $e_{n}(T: X \rightarrow Y), n=1,2, \ldots$, of bounded linear operators $T \in \mathcal{L}(X, Y)$ between quasi-Banach spaces are a well-established field of research (see, for example, the monographs $[\mathbf{6}, \mathbf{8}, \mathbf{2 1}, \mathbf{3 3}])$. The decay of the numbers $e_{n}(T: X \rightarrow Y)$ describes the compactness of $T$ in a qualitative way. In particular, $T$ is compact if and only if

$$
\lim _{n \rightarrow \infty} e_{n}(T: X \rightarrow Y)=0 .
$$

In this article we study the entropy numbers of identity operators

$$
\text { id }: B_{p_{1}, q_{1}}^{s_{1}}\left(\mathbb{R}^{d}, w_{1}\right) \rightarrow B_{p_{2}, q_{2}}^{s_{2}}\left(\mathbb{R}^{d}, w_{2}\right)
$$

mapping a weighted Besov space into another. In the case

$$
\frac{w_{1}(x)}{w_{2}(x)}=\left(1+|x|^{2}\right)^{\alpha / 2}
$$


this problem has a certain history, for which we refer to $[\mathbf{8}]$. The interesting phenomenon observed in this situation is the following. There is an interplay between the difference $\delta$ of the differential dimensions of the involved spaces

$$
\delta:=\left(s_{1}-\frac{d}{p_{1}}\right)-\left(s_{2}-\frac{d}{p_{2}}\right)
$$

and the ratio of the weights, represented by the power $\alpha$. In fact, it is known that

$$
e_{n}\left(\mathrm{id}: B_{p_{1}, q_{1}}^{s_{1}}\left(\mathbb{R}^{d}, w_{1}\right) \rightarrow B_{p_{2}, q_{2}}^{s_{2}}\left(\mathbb{R}^{d}, w_{2}\right)\right) \sim n^{-\min (\alpha, \delta) / d-1 / p_{1}+1 / p_{2}}
$$

as long as

$$
\delta \neq \alpha \quad \text { and } \quad \min (\alpha, \delta)>d \max \left(0, \frac{1}{p_{2}}-\frac{1}{p_{1}}\right)
$$

(see $[\mathbf{8}, \mathbf{1 5}, \mathbf{1 6}, \mathbf{2 3}])$. Here we present a new approach to this problem for a much more general class of weights. There are two different types of condition that our weights have to satisfy. One set of restrictions originates from the definition of the weighted Besov spaces and our method of discretization. We do not want to go into the details of this question here, instead we simply looked for the most convenient reference. A second set of restrictions is connected with our conditions on the weight functions, which will be expressed in terms of the upper and lower indices of the ratio $w_{1} / w_{2}$. These indices describe the behaviour of the function near infinity in some qualitative way. Under certain restrictions, the asymptotic behaviour of the entropy numbers is given by

$$
e_{n}\left(\mathrm{id}: B_{p_{1}, q_{1}}^{s_{1}}\left(\mathbb{R}^{d}, w_{1}\right) \rightarrow B_{p_{2}, q_{2}}^{s_{2}}\left(\mathbb{R}^{d}, w_{2}\right)\right) \sim n^{-1 / p_{1}+1 / p_{2}} \frac{w_{2}\left(n^{1 / d}, \ldots, n^{1 / d}\right)}{w_{1}\left(n^{1 / d}, \ldots, n^{1 / d}\right)}
$$

(roughly speaking, if $w_{1} / w_{2}$ is growing not too fast near infinity) or

$$
e_{n}\left(\mathrm{id}: B_{p_{1}, q_{1}}^{s_{1}}\left(\mathbb{R}^{d}, w_{1}\right) \rightarrow B_{p_{2}, q_{2}}^{s_{2}}\left(\mathbb{R}^{d}, w_{2}\right)\right) \sim n^{-\delta / d-1 / p_{1}+1 / p_{2}}
$$

(if $w_{1} / w_{2}$ is growing fast enough near infinity).

Our main tools for proving the estimate from above in (1.3) and (1.4), respectively, will be the use of operator ideals (see $[\mathbf{6}, \mathbf{2 1}, \mathbf{3 3}]$ ) and the basic estimates of entropy numbers $e_{n}\left(\mathrm{id}: \ell_{p_{1}}^{N} \rightarrow \ell_{p_{2}}^{N}\right)$ due to Schütt, Triebel, Edmunds and Kühn.

The paper is organized as follows. In $\S 2$ we recall the definition of the weighted Besov spaces and discuss a few of their properties. There are several standard techniques for relating Besov spaces to sequence spaces - we will use a characterization by wavelets. This enables us to deal with weighted sequence spaces instead of weighted function spaces. In $\S 3$ we give a new elementary proof of the characterization of those parameters for which a continuous (or compact, respectively) embedding exists. Section 4 represents the heart of the paper; here we investigate entropy numbers of embeddings of weighted sequence spaces. In $\S 4.1$ we recall some basic properties of operator ideals. Section 4.2 is devoted to the investigation of a certain set of weight functions and in $\S 4.3$ we prove our main result. Several examples are investigated in $\S 4.4$. Finally, in $\S 5$ we shift these results from the sequence space level to the function spaces and formulate some open problems. 


\section{Notation}

The symbol 'id' always refers to an identity operator. If it is clear from the context, we do not indicate the spaces between which id is considered, and likewise for other operators. Let $T$ be a linear operator which maps the quasi-Banach space $X$ into the quasi-Banach space $B$. If no confusion is possible, we feel free to write $\|T\|$ instead of the more exact versions $\|T \mid \mathcal{L}(X, Y)\|$ or $\|T: X \rightarrow Y\|$. We define $a \asymp b$ if there exists a constant $c>0$ (independent of the context-dependent relevant parameters) such that

$$
c^{-1} a \leqslant b \leqslant c a .
$$

All unimportant constants will be denoted by $c$, sometimes with additional indices.

\section{Agreement}

If no further restrictions are stated, then the parameters $p, p_{1}, p_{2}, q, q_{1}$ and $q_{2}$ may vary in $(0, \infty]$ and the parameters $s, s_{1}$ and $s_{2}$ in $\mathbb{R}$.

\section{Weighted Besov spaces}

In this section we recall a few of the properties of weighted Besov spaces.

\subsection{Definition and preliminaries}

We are interested in compact embeddings of weighted Besov spaces into unweighted ones. In such a situation it seems to be natural to concentrate on the behaviour of the weight near infinity and to ignore local singularities of the weight. So we restrict ourselves to smooth weights. For our purposes it will be convenient to work with the following class $\mathcal{W}_{1}$ of weights (see $[8]$ ).

Definition 2.1. We say that a function $w: \mathbb{R}^{d} \rightarrow(0, \infty)$ belongs to $\mathcal{W}_{1}$ if it satisfies the following conditions.

(i) The function $w$ is infinitely differentiable.

(ii) There exist a constant $c>0$ and a number $\alpha \geqslant 0$ such that

$$
0<w(x) \leqslant c w(y)(1+|x-y|)^{\alpha}
$$

holds for all $x, y \in \mathbb{R}^{d}$.

(iii) For all multi-indices $\alpha \in \mathbb{N}_{0}^{d}$ the quantities

$$
c_{w, \alpha}:=\sup _{x \in \mathbb{R}^{d}} \frac{\left|D^{\alpha} w(x)\right|}{w(x)}
$$

are finite.

Remark 2.2. Restriction (ii) implies that the weight grows at most polynomially near infinity. As will become clear later on, weights that increase faster are not interesting in our context (see Remark 5.17). 
For the definition of the (unweighted) Besov spaces we refer to the monographs [31, 41, 42]. As usual, $\mathcal{S}^{\prime}\left(\mathbb{R}^{d}\right)$ denotes the collection of all tempered distributions. For us it will be convenient to introduce weighted Lebesgue and weighted Besov spaces as follows.

Definition 2.3. Let $w \in \mathcal{W}_{1}$.

(i) We define $L_{p}\left(\mathbb{R}^{d}, w\right)$ to be the set of all Lebesgue-measurable functions $f$ such that $w f \in L_{p}\left(\mathbb{R}^{d}\right)$. The norm is then given by

$$
\left\|f\left|L_{p}\left(\mathbb{R}^{d}, w\right)\|=\| f w\right| L_{p}\left(\mathbb{R}^{d}\right)\right\| .
$$

(ii) The space $B_{p, q}^{s}\left(\mathbb{R}^{d}, w\right)$ is the collection of all tempered distributions $f \in \mathcal{S}^{\prime}\left(\mathbb{R}^{d}\right)$ such that $f w \in B_{p, q}^{s}\left(\mathbb{R}^{d}\right)$. We put

$$
\left\|f\left|B_{p, q}^{s}\left(\mathbb{R}^{d}, w\right)\|=\| f w\right| B_{p, q}^{s}\left(\mathbb{R}^{d}\right)\right\| .
$$

Remark 2.4. If no confusion is possible, then we drop $\mathbb{R}^{d}$ from the notation.

Remark 2.5. There are different ways to introduce weighted Besov spaces (see, for example, $[\mathbf{4 0}],[\mathbf{2 9}],[\mathbf{4}],[\mathbf{1 0}],[\mathbf{3 6}],[\mathbf{5}],[\mathbf{3 7}],[\mathbf{3 8}]$ or $[\mathbf{3 5}])$. In all these references the definitions are Fourier analytic. For the class $\mathcal{W}_{1}$ these different approaches coincide (see $[10,29,35-38])$.

Remark 2.6. An alternative way to introduce Besov spaces with weights is as follows. For a natural number $M$, a weight function $w$ and $f \in L_{p}\left(\mathbb{R}^{d}, w\right)$ we put

$$
\omega_{M}(f, t, w)_{p}:=\sup _{|h|<t}\left\|\Delta_{h}^{M} f \mid L_{p}\left(\mathbb{R}^{d}, w\right)\right\|, \quad t>0 .
$$

Let $0<s<M$ and $0<p, q \leqslant \infty$. Parallel to the unweighted case one defines $f \in$ $\Lambda_{p, q}^{s}\left(\mathbb{R}^{d}, w\right)$ if and only if $f \in L_{p}\left(\mathbb{R}^{d}, w\right)$ and

$$
\left(\int_{0}^{1}\left[t^{-s} \omega_{M}(f, t, w)_{p}\right]^{q} \frac{\mathrm{d} t}{t}\right)^{1 / q}<\infty
$$

Under the restrictions $w \in \mathcal{W}_{1}$ and $s>d \max (0,1 / p-1)$ the spaces $\Lambda_{p, q}^{s}\left(\mathbb{R}^{d}, w\right)$ and $B_{p, q}^{s}\left(\mathbb{R}^{d}, w\right)$ coincide (with equivalent norms). For $s<d \max (0,1 / p-1)$ the classes $B_{p, q}^{s}\left(\mathbb{R}^{d}, w\right)$ contain singular distributions, and hence they are different from $\Lambda_{p, q}^{s}\left(\mathbb{R}^{d}, w\right)$. We do not have a direct reference for the claimed coincidence. In [36, Theorem 5.1.4] a characterization by differences is proved. However, some standard manipulations based on maximal functions of Peetre-Fefferman-Stein type (see, for example, $[\mathbf{3 6}, 5.1]$ ) combined with the identification of $B_{p, q}^{s}\left(\mathbb{R}^{d}, w\right)$ with the above-mentioned Fourier-analytic counterpart yield the result.

Remark 2.7. Our definition has some advantages, e.g. embeddings carry over from the unweighted to the weighted case. 


\subsection{Besov spaces and sequence spaces}

We use the discrete wavelet transform for discretizing the quasi-norms in the weighted Besov spaces. First of all we need to fix some notation. The symbol ' $\hookrightarrow$ ' is used for continuous embeddings. By $\mathbb{N}$ we denote the set of natural numbers, by $\mathbb{N}_{0}$ the set $\mathbb{N} \cup\{0\}$, and by $\mathbb{Z}^{d}$ the set of all lattice points in $\mathbb{R}^{d}$ having integer coordinates.

Let $\tilde{\phi}$ be an orthogonal scaling function on $\mathbb{R}$ with compact support and of sufficiently high regularity. Let $\tilde{\psi}$ be a corresponding wavelet. Then the tensor product ansatz yields a scaling function $\phi$ and associated wavelets $\psi_{1}, \ldots, \psi_{2^{d}-1}$, now all defined on $\mathbb{R}^{d}$ and having compact support. Let $\tilde{\phi} \in C^{N}(\mathbb{R})$; then $\phi, \psi_{i} \in C^{N}\left(\mathbb{R}^{d}\right)$ as well. We will use the standard abbreviations

$$
\phi_{j, \ell}(x)=2^{j d / 2} \phi\left(2^{j} x-\ell\right) \quad \text { and } \quad \psi_{i, j, \ell}(x)=2^{j d / 2} \psi_{i}\left(2^{j} x-\ell\right) .
$$

Proposition 2.8. Let $w \in \mathcal{W}_{1}$. Suppose $N>\max \left(s,(2 d / p)+\frac{1}{2} d-s\right)$. Then a distribution $f \in \mathcal{S}^{\prime}$ belongs to $B_{p, q}^{s}(w)$ if and only if

$$
\begin{aligned}
\left\|f \mid B_{p, q}^{s}(w)\right\|^{\boldsymbol{*}}= & \left(\sum_{\ell \in \mathbb{Z}^{d}}\left|\left\langle f, \phi_{0, \ell}\right\rangle w(\ell)\right|^{p}\right)^{1 / p} \\
& +\sum_{i=1}^{2^{d}-1}\left\{\sum_{j=0}^{\infty} 2^{j(s+d(1 / 2-1 / p)) q}\left(\sum_{\ell \in \mathbb{Z}^{d}}\left|\left\langle f, \psi_{i, j, \ell}\right\rangle w\left(2^{-j} \ell\right)\right|^{p}\right)^{q / p}\right\}^{1 / q}<\infty .
\end{aligned}
$$

Furthermore, $\left\|f \mid B_{p, q}^{s}(w)\right\|^{\boldsymbol{*}}$ is an equivalent quasi-norm on $B_{p, q}^{s}(w)$.

Remark 2.9. A proof of this proposition may be found in [17]. But it has several forerunners in the Banach space situation (i.e. $p, q \geqslant 1$ ). We refer to $[\mathbf{3 0}],[\mathbf{4 5}]$ and $[\mathbf{2}]$. Further we mention [26], see also [19, II.6.4], [11] and [34], which are primarily interested in the homogeneous spaces. In this context they worked with more general weights.

\section{The characterization of continuous and compact embeddings of weighted sequence spaces}

The aim of this section is to characterize all constellations of parameters where either a continuous embedding or a compact embedding exists. By Proposition 2.8 we can do this on the side of the sequence spaces.

\subsection{Some preliminaries}

Motivated by Proposition 2.8 we introduce the following sequence spaces. For a given function $w: \mathbb{R}^{d} \rightarrow(0, \infty)$ we define

$$
\begin{aligned}
\ell_{q}\left(2^{j s} \ell_{p}(w)\right):=\{\lambda & =\left\{\lambda_{j, k}\right\}_{j, k}: \lambda_{j, k} \in \mathbb{C}, \\
& \left.\left\|\lambda \mid \ell_{q}\left(2^{j s} \ell_{p}(w)\right)\right\|=\left(\sum_{j=0}^{\infty} 2^{j s q}\left(\sum_{k \in \mathbb{Z}^{d}}\left|\lambda_{j, k} w\left(2^{-j} k\right)\right|^{p}\right)^{q / p}\right)^{1 / q}<\infty\right\}
\end{aligned}
$$


(with the usual modifications if $p=\infty$ and/or $q=\infty$ ). Hence, for the question of embeddings we may concentrate on the spaces $\ell_{q}\left(2^{j s} \ell_{p}(w)\right)$. Let $w_{1}, w_{2}: \mathbb{R}^{d} \rightarrow(0, \infty)$ be given functions. Observe that

$$
\ell_{q_{1}}\left(2^{j\left(s_{1}+d\left(1 / 2-1 / p_{1}\right)\right)} \ell_{p_{1}}\left(w_{1}\right)\right) \hookrightarrow \ell_{q_{2}}\left(2^{j\left(s_{2}+d\left(1 / 2-1 / p_{2}\right)\right)} \ell_{p_{2}}\left(w_{2}\right)\right)
$$

is equivalent to

$$
\ell_{q_{1}}\left(2^{j\left(s_{1}-s_{2}+d\left(1 / p_{2}-1 / p_{1}\right)\right)} \ell_{p_{1}}\left(w_{1} / w_{2}\right)\right) \hookrightarrow \ell_{q_{2}}\left(\ell_{p_{2}}\right) .
$$

So it is sufficient to consider unweighted spaces as target spaces.

\subsection{Continuous and compact embeddings of weighted sequence spaces}

We switch to a general point of view, picked up from [28]. Let $\beta=\left\{\beta_{j}\right\}_{j=0}^{\infty}$ and $w=\left\{w_{j, k}\right\}_{j, k}$ be sequences of positive numbers. Then we put

$$
\begin{aligned}
\ell_{q}\left(\beta_{j} \ell_{p}(w)\right):=\left\{\lambda=\left\{\lambda_{j, k}\right\}_{j, k}: \lambda_{j, k} \in \mathbb{C},\right. \\
\left.\left\|\lambda \mid \ell_{q}\left(\beta_{j} \ell_{p}(w)\right)\right\|=\left(\sum_{j=0}^{\infty} \beta_{j}^{q}\left(\sum_{k \in \mathbb{Z}^{d}}\left|\lambda_{j, k} w_{j, k}\right|^{p}\right)^{q / p}\right)^{1 / q}<\infty\right\}
\end{aligned}
$$

(with the usual modifications if $p=\infty$ and/or $q=\infty$ ). For a real number $a$ we define $a_{+}:=\max (a, 0)$.

\section{Theorem 3.1.}

(i) There is an embedding $\ell_{q_{1}}\left(\beta_{j} \ell_{p_{1}}(w)\right) \hookrightarrow \ell_{q_{2}}\left(\ell_{p_{2}}\right)$ if and only if

$$
\left\{\beta_{j}^{-1}\left\|\left\{\left(w_{j, k}\right)^{-1}\right\}_{k} \mid \ell_{p *}\right\|\right\}_{j} \in \ell_{q *},
$$

where

$$
\frac{1}{p^{*}}:=\left(\frac{1}{p_{2}}-\frac{1}{p_{1}}\right)_{+} \quad \text { and } \quad \frac{1}{q^{*}}:=\left(\frac{1}{q_{2}}-\frac{1}{q_{1}}\right)_{+} .
$$

Moreover, the following expression holds:

$$
\| \text { id }: \ell_{q_{1}}\left(\beta_{j} \ell_{p_{1}}(w)\right) \rightarrow \ell_{q_{2}}\left(\ell_{p_{2}}\right)\|=\|\left\{\beta_{j}^{-1}\left\|\left\{\left(w_{j, k}\right)^{-1}\right\}_{k} \mid \ell_{p *}\right\|\right\}_{j} \mid \ell_{q *} \| .
$$

(ii) The embedding $\ell_{q_{1}}\left(\beta_{j} \ell_{p_{1}}(w)\right) \hookrightarrow \ell_{q_{2}}\left(\ell_{p_{2}}\right)$ is compact if and only if

$$
\left\{\beta_{j}^{-1}\left(w_{j, k}\right)^{-1}\right\}_{j, k} \in \ell_{q^{*}}\left(\ell_{p *}\right),
$$

and in addition

$$
\lim _{j \rightarrow \infty} \beta_{j}^{-1}\left\|\left\{\left(w_{j, k}\right)^{-1}\right\}_{k} \mid \ell_{p *}\right\|=0 \quad \text { if } q^{*}=\infty
$$

and

$$
\lim _{|k| \rightarrow \infty} w_{j, k}=\infty \quad \text { for all } j \in \mathbb{N}_{0} \text { if } p^{*}=\infty
$$




\section{Proof.}

Step 1. Proof of (i).

The proof of the sufficiency of the condition $\left\{\beta_{j}^{-1}\left\|\left\{\left(w_{j, k}\right)^{-1}\right\}_{k} \mid \ell_{p *}\right\|\right\}_{j} \in \ell_{q *}$ is a simple exercise, using Hölder's inequality together with the monotonicity of the $\ell_{r}$-spaces with respect to $r$. So we concentrate on the proof of the necessity.

Substep 1.1. Let $p^{*}=\infty$ and assume that there exists a non-negative integer $j^{*}$ such that $\left\|\left\{\left(w_{j^{*}, k}\right)^{-1}\right\}_{k} \mid \ell_{\infty}\right\|=\infty$. Hence there is a sequence $\left\{k_{\nu}\right\}_{\nu=1}^{\infty} \subset \mathbb{Z}^{d}$ such that

$$
\lim _{\nu \rightarrow \infty}\left(w_{j^{*}, k_{\nu}}\right)^{-1}=\infty
$$

We define a sequence of elements in $\ell_{q_{1}}\left(\beta_{j} \ell_{p_{1}}(w)\right)$ by

$$
\left(\lambda^{\nu}\right)_{j, k}:= \begin{cases}\left(w_{j^{*}, k_{\nu}}\right)^{-1} & \text { if } j=j^{*} \text { and } k=k_{\nu} \\ 0 & \text { otherwise. }\end{cases}
$$

Then

$$
\left\|\lambda^{\nu} \mid \ell_{q_{1}}\left(\beta_{j} \ell_{p_{1}}(w)\right)\right\|=\beta_{j^{*}}, \quad \nu \in \mathbb{N}
$$

and

$$
\left\|\lambda^{\nu} \mid \ell_{q_{2}}\left(\ell_{p_{2}}\right)\right\|=\left(w_{j^{*}, k_{\nu}}\right)^{-1}, \quad \nu \in \mathbb{N} .
$$

Letting $\nu \rightarrow \infty$, this contradicts the embedding $\ell_{q_{1}}\left(\beta_{j} \ell_{p_{1}}(w)\right) \hookrightarrow \ell_{q_{2}}\left(\ell_{p_{2}}\right)$. Hence $\left\|\left\{\left(w_{j, k}\right)^{-1}\right\}_{k} \mid \ell_{\infty}\right\|<\infty$ for all $j \in \mathbb{N}_{0}$.

Substep 1.2. Let $p^{*}<\infty$ and assume that there exists a non-negative integer $j^{*}$ such that $\left\|\left\{\left(w_{j^{*}, k}\right)^{-1}\right\}_{k} \mid \ell_{p *}\right\|=\infty$. Then there exists a sequence $\left\{\ell_{\nu}\right\}_{\nu=1}^{\infty} \subset \mathbb{N}$ such that

$$
\sum_{|k| \leqslant 2^{\ell_{\nu}-1}}\left(w_{j^{*}, k}\right)^{-p^{*}}+2^{\nu} \leqslant \sum_{|k| \leqslant 2^{\ell_{\nu}}}\left(w_{j^{*}, k}\right)^{-p^{*}} .
$$

To shorten the notation we put

$$
a_{1}:=\sum_{|k| \leqslant 2^{\ell_{1}}}\left(w_{j^{*}, k}\right)^{-p^{*}} \text { and } a_{\nu}:=\sum_{2^{\ell_{\nu}-1}<|k| \leqslant 2^{\ell_{\nu}}}\left(w_{j^{*}, k}\right)^{-p^{*}}, \quad \nu \geqslant 2 .
$$

Defining, moreover,

$$
\lambda_{j, k}:= \begin{cases}\left(w_{j^{*}, k}\right)^{-p^{*} / p_{2}} a_{\nu}^{-1 / p_{2}} & \text { if } j=j^{*}, 2^{\ell_{\nu-1}}<|k| \leqslant 2^{\ell_{\nu}} \text { and } \nu \geqslant 2 \\ 0 & \text { or }|k| \leqslant 2^{\ell_{1}} \text { and } \nu=1 \\ & \text { otherwise }\end{cases}
$$

we get

$$
\left\|\lambda \mid \ell_{q_{2}}\left(\ell_{p_{2}}\right)\right\|=\infty
$$


Let $p_{1}<\infty$. Due to the fact that

$$
\begin{aligned}
\sum_{\nu=2}^{\infty} \sum_{2^{\ell_{\nu}-1}<|k| \leqslant 2^{\ell_{\nu}}}\left(\left(w_{j^{*}, k}\right)^{-p^{*} / p_{2}} a_{\nu}^{-1 / p_{2}} w_{j^{*}, k}\right)^{p_{1}} & \\
& =\sum_{\nu=2}^{\infty} a_{\nu}^{-p_{1} / p_{2}} \sum_{2^{\ell_{\nu-1}<|k| \leqslant 2^{\ell_{\nu}}}}\left(w_{j^{*}, k}\right)^{p_{1}-\left(p_{1} p^{*}\right) / p_{2}} \\
& =\sum_{\nu=2}^{\infty} a_{\nu}^{1-p_{1} / p_{2}} \\
& \leqslant \sum_{\nu=2}^{\infty} 2^{\nu\left(1-p_{1} / p_{2}\right)}<\infty
\end{aligned}
$$

where we used

$$
a_{\nu} \geqslant 2^{\nu}, \quad \nu \geqslant 2 \text { and } p_{2}<p_{1},
$$

it follows that $\left\|\lambda \mid \ell_{q_{1}}\left(\beta_{j} \ell_{p_{1}}(w)\right)\right\|<\infty$. But this contradicts the embedding

$$
\ell_{q_{1}}\left(\beta_{j} \ell_{p_{1}}(w)\right) \hookrightarrow \ell_{q_{2}}\left(\ell_{p_{2}}\right) .
$$

Hence $\left\|\left\{\left(w_{j, k}\right)^{-1}\right\}_{k} \mid \ell_{p^{*}}\right\|<\infty$ for all $j \in \mathbb{N}_{0}$ The case $p_{1}=\infty$ can be treated similarly.

Substep 1.3. To finish the proof of (i) we exploit the fact that the best constant $c$ in

$$
\left\|\tau_{k}\left|\ell_{p_{2}}\|\leqslant c\|\left\{\left(w_{j, k}\right)^{-1}\right\}_{k}\right| \ell_{p^{*}}\right\|\left\|\tau_{k} \mid \ell_{p_{1}}(w)\right\|, \quad\left\{\tau_{k}\right\}_{k} \in \ell_{p_{1}}(w),
$$

is $c=1$. Hence, for all $\varepsilon>0$ and all $j \in \mathbb{N}_{0}$ there exists a sequence $\left\{\tau_{j, k}\right\}_{k} \in \ell_{p_{1}}(w)$ such that $\left\|\left\{\tau_{j, k}\right\}_{k} \mid \ell_{p_{2}}\right\|=1$ and

$$
\left\|\left\{\tau_{j, k}\right\}_{k}\left|\ell_{p_{2}}\left\|\geqslant\left(1-2^{-j} \varepsilon\right)\right\|\left\{\left(w_{j, k}\right)^{-1}\right\}_{k}\right| \ell_{p^{*}}\right\|\left\|\left\{\tau_{j, k}\right\}_{k} \mid \ell_{p_{1}}(w)\right\| .
$$

We put

$$
\mu_{j}:=\beta_{j}^{-1}\left\|\left\{\left(w_{j, k}\right)^{-1}\right\}_{k} \mid \ell_{p^{*}}\right\| \quad \text { and } \quad \nu_{j}:=\beta_{j}\left\|\left\{\tau_{j, k}\right\}_{k} \mid \ell_{p_{1}}(w)\right\|, \quad j \in \mathbb{N}_{0} .
$$

By Substeps 1.1 and 1.2 we know that the $\mu_{j}$ are finite. Then

$$
\left\|\left\{\tau_{j, k}\right\}_{k} \mid \ell_{p_{2}}\right\| \geqslant\left(1-2^{-j} \varepsilon\right) \mu_{j} \nu_{j} .
$$

By the same reasoning as before we obtain that for every $\varepsilon>0$ there exists a sequence $\left\{\gamma_{j}\right\}_{j}$ such that $\left\|\left\{\gamma_{j}\right\}_{j} \mid \ell_{q_{2}}\right\|=1$ and

$$
\left\|\left\{\gamma_{j}\right\}_{j}\left|\ell_{q_{2}}\|\geqslant(1-\varepsilon)\|\left\{\mu_{j}\right\}_{j}\right| \ell_{q^{*}}\right\|\left\|\left\{\mu_{j}^{-1} \gamma_{j}\right\}_{j} \mid \ell_{q_{1}}\right\| .
$$

Setting $\delta_{j}:=\gamma_{j} /\left(\mu_{j} \nu_{j}\right)$ for $j \in \mathbb{N}_{0}$, we arrive at

$$
\begin{aligned}
\left(\sum_{j=0}^{M}\left(\delta_{j}\left\|\left\{\tau_{j, k}\right\}_{k} \mid \ell_{p_{2}}\right\|\right)^{q_{2}}\right)^{1 / q_{2}} & \geqslant(1-\varepsilon)\left\|\left\{\delta_{j} \mu_{j} \nu_{j}\right\}_{j} \mid \ell_{q_{2}}\right\| \\
& \geqslant(1-\varepsilon)^{2}\left\|\left\{\mu_{j}\right\}_{j}\left|\ell_{q^{*}}\|\|\left\{\delta_{j} \nu_{j}\right\}_{j}\right| \ell_{q_{1}}\right\| \\
& \geqslant(1-\varepsilon)^{2}\left\|\left\{\mu_{j}\right\}_{j}\left|\ell_{q^{*}}\|\|\left\{\delta_{j} \tau_{j, k}\right\}_{j, k}\right| \ell_{q_{1}}\left(\beta_{j} \ell_{p_{1}}(w)\right)\right\|
\end{aligned}
$$

if $M$ is chosen sufficiently large. This proves the claim. 
Step 2. Proof of (ii).

Substep 2.1. Proof of sufficiency. We are going to prove that, under the given restrictions in (ii), the identity id $: \ell_{q_{1}}\left(\beta_{j} \ell_{p_{1}}(w)\right) \rightarrow \ell_{q_{2}}\left(\ell_{p_{2}}\right)$ is the limit (in the operator norm) of a sequence of finite-rank operators. To this end we introduce a certain decomposition of the identity which will also be of great use to us later. Let

$$
\Lambda:=\left\{\lambda=\left(\lambda_{j, k}\right)_{j \in \mathbb{N}_{0}, k \in \mathbb{Z}^{d}}: \lambda_{j, k} \in \mathbb{C}, j \in \mathbb{N}_{0}, k \in \mathbb{Z}^{d}\right\},
$$

and consider the index sets $I_{j, i} \subset \mathbb{N}_{0} \times \mathbb{Z}^{d}$ given by

$$
\begin{aligned}
I_{j, 0} & :=\left\{(j, k):|k| \leqslant 2^{j}\right\}, \quad j \in \mathbb{N}_{0}, \\
I_{j, i} & :=\left\{(j, k): 2^{j+i-1}<|k| \leqslant 2^{j+i}\right\}, \quad i \in \mathbb{N}, j \in \mathbb{N}_{0} .
\end{aligned}
$$

Furthermore, let $P_{j, i}: \Lambda \rightarrow \Lambda$ be the canonical projection onto the coordinates in $I_{j, i}$, i.e. for $\lambda \in \Lambda$ we put

$$
\left(P_{j, i} \lambda\right)_{u, v}:=\left\{\begin{array}{ll}
\lambda_{u, v} & (u, v) \in I_{j, i}, \\
0 & \text { otherwise }
\end{array} \quad u \in \mathbb{N}_{0}, v \in \mathbb{Z}^{d} .\right.
$$

Observe that

$$
M_{j, i}:=\left|I_{j, i}\right| \sim 2^{(j+i) d},
$$

and

$$
\operatorname{id}_{\Lambda}=\sum_{j=0}^{\infty} \sum_{i=0}^{\infty} P_{j, i} .
$$

For all natural numbers $J$ and $M$ and $j \in \mathbb{N}_{0}$, we set

$$
P_{J}:=\sum_{j=J}^{\infty} \sum_{i=0}^{\infty} P_{j, i} \quad \text { and } \quad Q_{j, M}:=\sum_{i=M+1}^{\infty} P_{j, i} .
$$

Hölder's inequality yields

$$
\left\|P_{J} \lambda\left|\ell_{q_{2}}\left(\ell_{p_{2}}\right)\|\leqslant\|\left\{\beta_{j}^{-1}\left\|\left\{\left(w_{j, k}\right)^{-1}\right\}_{k} \mid \ell_{p *}\right\|\right\}_{j=J}^{\infty}\right| \ell_{q^{*}}\right\|\left\|\lambda \mid \ell_{q_{1}}\left(\beta_{j} \ell_{p_{1}}(w)\right)\right\| .
$$

By assumption (3.2), for any $\varepsilon>0$ there exists some $J$ such that

$$
\left\|\left\{\beta_{j}^{-1}\left\|\left\{\left(w_{j, k}\right)^{-1}\right\}_{k} \mid \ell_{p *}\right\|\right\}_{j=J}^{\infty} \mid \ell_{q^{*}}\right\|<\varepsilon .
$$

Furthermore, for any sequence $\left\{M_{j}\right\}_{j}$ of natural numbers we have

$$
\begin{aligned}
\left\|\sum_{j=0}^{J-1} Q_{j, M_{j}} \lambda \mid \ell_{q_{2}}\left(\ell_{p_{2}}\right)\right\| & \\
& \leqslant\left\|\left\{\beta_{j}^{-1}\left(\sum_{|k|>2^{j+M_{j}}}\left|\left(w_{j, k}\right)^{-1}\right|^{p^{*}}\right)^{1 / p *}\right\}_{j=0}^{J-1}\left|\ell_{q^{*}}\|\| \lambda\right| \ell_{q_{1}}\left(\beta_{j} \ell_{p_{1}}(w)\right)\right\| .
\end{aligned}
$$


We put $\beta:=\beta(J)=\left\|\left\{\beta_{j}^{-1}\right\}_{j=0}^{J-1} \mid \ell_{q^{*}}\right\|$. Because of (3.3) we have

$$
\left\|\left\{\left(w_{j, k}\right)^{-1}\right\}_{|k|>2^{j+M_{j}}} \mid \ell_{p *}\right\| \leqslant \beta^{-1} \varepsilon
$$

if all numbers $M_{j}$ are chosen sufficiently large. Hence, for those $M_{j}$ we obtain

$$
\left\|\operatorname{id}_{\Lambda}-\sum_{j=0}^{J-1} \sum_{i=0}^{M_{j}} P_{j, i} \mid \ell_{q_{1}}\left(\beta_{j} \ell_{p_{1}}(w)\right) \rightarrow \ell_{q_{2}}\left(\ell_{p_{2}}\right)\right\| \leqslant 2^{1 / \min \left(1, p^{*}, q^{*}\right)} \varepsilon .
$$

Since the range space of $\sum_{j=0}^{J-1} \sum_{i=0}^{M_{j}} P_{j, i}$ is obviously finite dimensional we are done.

Substep 2.2. Proof of necessity.

If $\max \left(q^{*}, p^{*}\right)<\infty$, necessity is already proved in (i).

Now let $p^{*}=\infty$. Assume that there are a number $j^{*} \in \mathbb{N}_{0}$ and a sequence $\left\{k_{\nu}\right\}_{\nu} \subset \mathbb{Z}^{d}$ such that

$$
\lim _{\nu \rightarrow \infty}\left|k_{\nu}\right|=\infty \text { and }\left(w_{j^{*}, k_{\nu}}\right)^{-1} \geqslant c>0 \text { for all } \nu .
$$

Now we use the same sequence $\lambda^{\nu}$ as in Step 1 (see (3.4)). Recall that for all $\nu \in \mathbb{N}$ it holds that $\left\|\lambda^{\nu} \mid \ell_{q_{1}}\left(\beta_{j} \ell_{p_{1}}(w)\right)\right\|=\beta_{j^{*}}$. But for $\nu \neq \mu$ we have

$$
\left\|\lambda^{\nu}-\lambda^{\mu} \mid \ell_{q_{2}}\left(\ell_{p_{2}}\right)\right\|=\left(\left(w_{j^{*}, k_{\nu}}\right)^{-p_{2}}+\left(w_{j^{*}, k_{\mu}}\right)^{-p_{2}}\right)^{1 / p_{2}} \geqslant c>0 .
$$

It remains to consider the case $q^{*}=\infty$. Let us assume that there exists a sequence $\left\{j_{\nu}\right\}_{\nu}$ of natural numbers tending to infinity and such that

$$
\beta_{j_{\nu}}^{-1}\left\|\left\{\left(w_{j_{\nu}, k}\right)^{-1}\right\}_{k} \mid \ell_{p^{*}}\right\| \geqslant c>0 \quad \text { for all } \nu .
$$

We define

$$
\left(\lambda^{\nu}\right)_{j, k}:= \begin{cases}\beta_{j_{\nu}}^{-1}\left\|\left\{\left(w_{j_{\nu}, k}\right)^{-1}\right\}_{k} \mid \ell_{p^{*}}\right\| & \text { if } j=j_{\nu} \text { and } k=(0, \ldots, 0) \\ 0 & \text { otherwise. }\end{cases}
$$

Then

$$
\left\|\lambda^{\nu}\left|\ell_{q_{1}}\left(\beta_{j} \ell_{p_{1}}(w)\right)\|=\|\left\{\left(w_{j_{\nu}, k}\right)^{-1}\right\}_{k}\right| \ell_{p^{*}}\right\| w_{j_{\nu},(0, \ldots, 0)} \leqslant 1, \quad \nu \in \mathbb{N} .
$$

But for $\nu \neq \mu$ we have

$$
\begin{aligned}
& \left\|\lambda^{\nu}-\lambda^{\mu} \mid \ell_{q_{2}}\left(\ell_{p_{2}}\right)\right\| \\
& \quad=\left(\beta_{j_{\nu}}^{-p_{2}}\left\|\left\{\left(w_{j_{\nu}, k}\right)^{-1}\right\}_{k}\left|\ell_{p^{*}}\left\|^{p_{2}}+\beta_{j_{\mu}}^{-p_{2}}\right\|\left\{\left(w_{j_{\mu}, k}\right)^{-1}\right\}_{k}\right| \ell_{p^{*}}\right\|^{p_{2}}\right)^{1 / p_{2}} \geqslant c>0 .
\end{aligned}
$$

This completes the proof.

Remark 3.2. The counterexample used in Substep 1.2 was brought to our attention by G. A. Kalyabin.

Remark 3.3. The decomposition of the identity (3.8) introduced in Substep 2.1 will also be of great service to us later on. It will be used in all estimates of the entropy numbers given in this article. 


\section{Entropy numbers of embeddings of weighted sequence spaces}

We are interested in measuring the compactness of the embeddings

$$
\text { id }: \ell_{q_{1}}\left(2^{j\left(s_{1}+d\left(1 / 2-1 / p_{1}\right)\right)} \ell_{p_{1}}\left(w_{1}\right)\right) \rightarrow \ell_{q_{2}}\left(2^{j\left(s_{2}+d\left(1 / 2-1 / p_{2}\right)\right)} \ell_{p_{2}}\left(w_{2}\right)\right) .
$$

Here $w_{1}, w_{2}: \mathbb{R}^{d} \rightarrow(0, \infty)$ are given functions that need not satisfy any further condition.

\subsection{Preliminaries}

Let us recall the definition of entropy numbers.

Definition 4.1. Let $X, Y$ be complex quasi-Banach spaces, let $T: X \rightarrow Y$ be a continuous linear operator, and let $n \in \mathbb{N}$. The $n$th entropy number $e_{n}(T: X \rightarrow Y)$ is the infimum of all numbers $\varepsilon>0$ such that there exist $2^{n-1}$ balls in $Y$ of radius $\varepsilon$ which cover the image of the closed unit ball $\left\{x \in X:\|x\|_{X} \leqslant 1\right\}$ under the mapping $T$.

In particular,

$$
T \text { is compact } \Longleftrightarrow \quad \lim _{n \rightarrow \infty} e_{n}(T)=0 .
$$

For details and basic properties we refer to the monographs $[\mathbf{6}, \mathbf{8}, \mathbf{2 1}, \mathbf{3 3}]$.

In what follows we will frequently use the abbreviations

$$
\delta:=s_{1}-s_{2}-d\left(\frac{1}{p_{1}}-\frac{1}{p_{2}}\right)
$$

and

$$
\frac{1}{p^{*}}:=\left(\frac{1}{p_{2}}-\frac{1}{p_{1}}\right)_{+} \text {and } \quad \frac{1}{q^{*}}:=\left(\frac{1}{q_{2}}-\frac{1}{q_{1}}\right)_{+} .
$$

For simplicity of notation we set

$$
B_{1}:=\ell_{q_{1}}\left(2^{j \delta} \ell_{p_{1}}\left(w_{1} / w_{2}\right)\right) \text { and } \quad B_{2}:=\ell_{q_{2}}\left(\ell_{p_{2}}\right) .
$$

Then the mapping $I$ defined by

$$
\lambda_{j, k} \stackrel{I}{\rightarrow} \lambda_{j, k} 2^{j\left(s_{2}+d\left(1 / 2-1 / p_{2}\right)\right)} w_{2}\left(2^{-j} k\right), \quad j \in \mathbb{N}_{0}, k \in \mathbb{Z}^{d},
$$

yields an isometry of

$$
\ell_{q_{1}}\left(2^{j\left(s_{1}+d\left(1 / 2-1 / p_{1}\right)\right)} \ell_{p_{1}}\left(w_{1}\right)\right)
$$

onto $\ell_{q_{1}}\left(2^{j \delta} \ell_{p_{1}}\left(w_{1} / w_{2}\right)\right)=B_{1}$. Furthermore, $I^{-1}$ yields an isometry of $\ell_{q_{2}}\left(\ell_{p_{2}}\right)=B_{2}$ onto

$$
\ell_{q_{2}}\left(2^{j\left(s_{2}+d\left(1 / 2-1 / p_{2}\right)\right)} \ell_{p_{2}}\left(w_{2}\right)\right)
$$

As a consequence of the definition of the entropy numbers and the properties of $I$ and $I^{-1}$ we obtain

$$
\begin{aligned}
e_{n}\left(\mathrm{id}: \ell_{q_{1}}\left(2^{j\left(s_{1}+d\left(1 / 2-1 / p_{1}\right)\right)} \ell_{p_{1}}\left(w_{1}\right)\right) \rightarrow \ell_{q_{2}}\left(2^{j\left(s_{2}+d\left(1 / 2-1 / p_{2}\right)\right)} \ell_{p_{2}}\left(w_{2}\right)\right)\right) & \\
& =e_{n}\left(\mathrm{id}: B_{1} \rightarrow B_{2}\right), \quad n=1,2, \ldots .
\end{aligned}
$$

Hence we may concentrate on $e_{n}\left(\right.$ id $\left.: B_{1} \rightarrow B_{2}\right)$. 


\subsection{Operator ideals}

The abstract concept of operator ideals has been proved to be a very useful tool in many different situations (see $[\mathbf{6}, \mathbf{3 2}, \mathbf{3 3}]$ ). Here it simplifies the estimates of the entropy numbers, and this observation has opened the door to the study of non-polynomial weights. Before we demonstrate this in the following subsections we need to extend this notion to operators acting between quasi-Banach spaces.

Let $E$ be a quasi-normed symmetric sequence space over the index set $\mathbb{N}$, i.e.

(i) $(1,0, \ldots) \in E$, in fact, we assume $\|(1,0, \ldots)\|_{E}=1$;

(ii) if $\left|a_{n}\right| \leqslant b_{n}$ for all $n$ and $b=\left(b_{1}, b_{2}, \ldots\right) \in E$, then $a \in E$ and $\|a\|_{E} \leqslant\|b\|_{E}$;

(iii) for each bijection $\pi: \mathbb{N} \rightarrow \mathbb{N}$ we have $\left\|\left(a_{1}, a_{2}, \ldots\right)\right\|_{E}=\left\|\left(a_{\pi(1)}, a_{\pi(2)}, \ldots\right)\right\|_{E}$.

$E$ is called maximal if

(i) $a \in E$ if and only if $\|a\|_{E}<\infty$;

(ii) $\|a\|_{E}=\sup _{n \in \mathbb{N}}\left\|P_{n} a\right\|_{E}$, where $P_{n} a=\left(a_{1}, a_{2}, \ldots, a_{n}, 0, \ldots\right)$.

Definition 4.2. Let $X, Y$ be quasi-Banach spaces and let $T \in \mathcal{L}(X, Y)$. Then we put

$$
L_{E}^{(e)}(T):=\left\|\left(e_{n}(T)\right)\right\|_{E}
$$

and

$$
\mathcal{L}_{E}^{(e)}(X, Y):=\left\{T \in \mathcal{L}(X, Y): L_{E}^{(e)}(T)<\infty\right\} .
$$

Theorem 4.3. Let $E$ be a maximal quasi-normed symmetric sequence space. Then $\mathcal{L}_{E}^{(e)}(X, Y)$ is a complete quasi-normed space, i.e. a quasi-Banach space. In particular, there exists an equivalent quasi-norm $\|\cdot\|_{*}$ on $\mathcal{L}_{E}^{(e)}(X, Y)$ and a number $0<\varrho \leqslant 1$ such that

$$
\left\|T_{1}+T_{2}\right\|_{*}^{\varrho} \leqslant\left\|T_{1}\right\|_{*}^{\varrho}+\left\|T_{2}\right\|_{*}^{\varrho} .
$$

Remark 4.4. The proof follows the same lines as the one for operators in Banach spaces - we refer to $[\mathbf{3 2}, 14.1 .8]$.

\subsection{A second class of weights}

For the entropy numbers of embeddings of weighted Besov spaces the behaviour of the weights near infinity is important. To describe this behaviour we will use indices, a well-known concept in analysis. First, we investigate functions defined on $[1, \infty)$.

Definition 4.5. We say that a measurable function $\varphi:[1, \infty) \rightarrow(0, \infty)$ belongs to $\mathcal{V}$ if the inequalities

$$
0<\underline{\varphi}(t):=\inf _{s \in[1, \infty)} \frac{\varphi(t s)}{\varphi(s)}, \quad \bar{\varphi}(t):=\sup _{s \in[1, \infty)} \frac{\varphi(t s)}{\varphi(s)}<\infty \text { for all } t \in[1, \infty),
$$

are satisfied and if $\underline{\varphi}$ and $\bar{\varphi}$ are measurable. 
Remark 4.6. Conditions of the above type have been used in several situations, e.g. in connection with real interpolation with a function parameter (see $[\mathbf{7 , 1 2}, \mathbf{2 0}, \mathbf{3 0}])$ or in the theory of function spaces with generalized smoothness (see $[\mathbf{3}, \mathbf{7}])$. However, in the quoted papers the functions have been defined either on $\mathbb{R}$ or on $(0, \infty)$.

Remark 4.7. Avakumovic, and independently Karamata, introduced and investigated a class $O R$ of functions which is more general than our one (see also $[\mathbf{1}, \S 2.0]$ ). We prefer to work with Definition 4.5.

For a function $\varphi \in \mathcal{V}$ the associated function $\bar{\varphi}$ is submultiplicative on $[1, \infty)$, i.e.

$$
\bar{\varphi}(s t) \leqslant \bar{\varphi}(s) \bar{\varphi}(t), \quad 1 \leqslant s, t<\infty .
$$

This implies that the function $s \mapsto \log \bar{\varphi}\left(\mathrm{e}^{s}\right)$ is subadditive on $(0, \infty)$. Theorems 6.4.1 and 6.6.1 in [18] guarantee that

$$
\alpha_{\varphi}:=\inf _{t>1} \frac{\log \bar{\varphi}(t)}{\log t}
$$

is either a real number or equal to $-\infty$. Furthermore, the function $1 / \underline{\varphi}$ is submultiplicative on $[1, \infty)$, too, and consequently the function $s \mapsto \log (1 / \underline{\varphi})\left(\mathrm{e}^{s}\right)$ is subadditive on $(0, \infty)$. Similarly as above this implies that

$$
\beta_{\varphi}:=\sup _{t>1} \frac{\log \underline{\varphi}(t)}{\log t}
$$

is either a real number or equal to $+\infty$. The obvious inequality $\beta_{\varphi} \leqslant \alpha_{\varphi}$ shows that both indices $\alpha_{\varphi}$ and $\beta_{\varphi}$ are real numbers. Moreover, by Theorem 6.4.1 in [18], a subadditive measurable function on $(0, \infty)$ is bounded on each compact subinterval of the real line. This leads to the following result.

Lemma 4.8. Suppose $\varphi \in \mathcal{V}$.

(i) For all $s \geqslant 1$ and $t \geqslant 1$ we have

$$
\underline{\varphi}(t) \varphi(s) \leqslant \varphi(t s) \leqslant \bar{\varphi}(t) \varphi(s) .
$$

(ii) The following inequality holds:

$$
-\infty<\beta_{\varphi} \leqslant \alpha_{\varphi}<\infty
$$

(iii) For any $\varepsilon>0$ there exists a constant $c_{\varepsilon} \geqslant 1$ such that

$$
c_{\varepsilon}^{-1} s^{\beta_{\varphi}-\varepsilon} \leqslant \underline{\varphi}(s) \leqslant \frac{\varphi(s)}{\varphi(1)} \leqslant \bar{\varphi}(s) \leqslant c_{\varepsilon} s^{\alpha_{\varphi}+\varepsilon} \quad \text { for all } s \geqslant 1 .
$$

As a consequence of this lemma we obtain some very useful estimates of certain sums appearing later in our estimates of the entropy numbers. 
Lemma 4.9. Let $\varrho>0$ and $\varphi \in \mathcal{V}$.

(i) If $\gamma>\alpha_{\varphi}$, then

$$
\sup _{M \geqslant 1} \varphi\left(2^{M}\right)^{\varrho} \sum_{m=0}^{M} 2^{-m \gamma \varrho} \varphi\left(2^{M-m}\right)^{-\varrho}<\infty .
$$

(ii) If $\gamma<\beta_{\varphi}$, then

$$
\sup _{M \geqslant 1} 2^{M \gamma \varrho} \sum_{m=0}^{M} 2^{-m \gamma \varrho} \varphi\left(2^{M-m}\right)^{-\varrho}<\infty
$$

and

$$
\sup _{M \geqslant 1} \varphi\left(2^{M}\right)^{\varrho} 2^{-M \gamma \varrho} \sum_{m=M+1}^{\infty} 2^{m \gamma \varrho} \varphi\left(2^{m}\right)^{-\varrho}<\infty .
$$

Proof.

Step 1. Proof of (i).

Making use of the second of the two inequalities in parts (i) and (iii) of Lemma 4.8 we find that

$$
\begin{aligned}
\varphi\left(2^{M}\right)^{\varrho} \sum_{m=0}^{M} 2^{-m \gamma \varrho} \varphi\left(2^{M-m}\right)^{-\varrho} & \leqslant \sum_{m=0}^{M} 2^{-m \gamma \varrho} \bar{\varphi}\left(2^{m}\right)^{\varrho} \\
& \leqslant c_{\varepsilon}^{\varrho} \sum_{m=0}^{M} 2^{m \varrho\left(-\gamma+\alpha_{\varphi}+\varepsilon\right)} \\
& \leqslant c_{\gamma, \alpha_{\varphi}}
\end{aligned}
$$

as long as $0<\varepsilon<\gamma-\alpha_{\varphi}$.

Step 2. We prove the second part of (ii). The proof of the first part is similar and therefore omitted. Applying the first of the two inequalities in parts (i) and (iii) of Lemma 4.8 we obtain

$$
\begin{aligned}
\varphi\left(2^{M}\right)^{\varrho} 2^{-M \gamma \varrho} \sum_{m=M+1}^{\infty} 2^{m \gamma \varrho} \varphi\left(2^{m}\right)^{-\varrho} & =\sum_{m=M+1}^{\infty} 2^{(m-M) \gamma \varrho} \varphi\left(2^{M}\right)^{\varrho} \varphi\left(2^{m}\right)^{-\varrho} \\
& \leqslant \sum_{m=M+1}^{\infty} 2^{(m-M) \gamma \varrho} \underline{\varphi}\left(2^{m-M}\right)^{-\varrho} \\
& \leqslant c_{\varepsilon}^{\varrho} \sum_{m=M+1}^{\infty} 2^{(m-M) \gamma \varrho} 2^{-(m-M)\left(\beta_{\varphi}-\varepsilon\right) \varrho} \\
& \leqslant c_{\varepsilon}^{\varrho} \sum_{m=M+1}^{\infty} 2^{(m-M)\left(\gamma-\beta_{\varphi}+\varepsilon\right) \varrho},
\end{aligned}
$$

which is finite if $\gamma-\beta_{\varphi}+\varepsilon<0$. 
The class of weights for which we are able to estimate the entropy numbers of the embeddings of sequence spaces is now given by the following definition.

Definition 4.10. A function $w: \mathbb{R}^{d} \rightarrow(0, \infty)$ belongs to the class $\mathcal{W}_{2}$ if there exist positive constants $c_{1}$ and $c_{2}$ and a function $\varphi \in \mathcal{V}$ such that

$$
\begin{aligned}
c_{1} & \leqslant w(x) \leqslant c_{2}, & & \text { for all } x,|x| \leqslant 1, \\
c_{1} \varphi(|x|) & \leqslant w(x) \leqslant c_{2} \varphi(|x|), & & \text { for all } x,|x|>1 .
\end{aligned}
$$

We say that a function $\varphi \in \mathcal{V}$ is 'associated with' $w$ if these inequalities are satisfied.

\subsection{Examples}

Before we list some examples, we consider a certain class of functions that will be of some use in this connection.

Lemma 4.11. Let $\eta$ and $\xi$ be real-valued, bounded and measurable functions, defined on $[1, \infty)$. We put

$$
\varphi(t):=\exp \left\{\eta(t)+\int_{1}^{t} \xi(u) \frac{\mathrm{d} u}{u}\right\}, \quad t \in[1, \infty)
$$

Then $\varphi \in \mathcal{V}$. Furthermore,

$$
\alpha_{\varphi}=\inf \sup _{t>1} \xi(t)
$$

where the infimum is taken over all possible representations (4.8) of $\varphi$, and

$$
\beta_{\varphi}=\sup \inf _{t>1} \xi(t)
$$

where the supremum is taken over the same set as before.

Proof. The proof of these assertions follows from a combination of Theorems 2.2.2 and 2.2.7 in [1] and Lemma 4.8 (iii).

\section{Polynomial weights}

Let $\alpha>0$. We put

$$
w_{\alpha}(x):=\left(1+|x|^{2}\right)^{\alpha / 2}, \quad x \in \mathbb{R}^{d} .
$$

Then $w_{\alpha} \in \mathcal{W}_{1} \cap \mathcal{W}_{2}$. An associated function $\varphi$ is given by $\varphi(x)=|x|^{\alpha}$, and a simple calculation yields $\alpha_{\varphi}=\beta_{\varphi}=\alpha$.

Small perturbations of polynomial weights

Let $\psi:[0, \infty) \rightarrow(0, \infty)$ be a positive and continuous function such that

$$
\psi(t)=\exp \left\{\int_{1}^{t} \varepsilon(u) \frac{\mathrm{d} u}{u}\right\}, \quad t \in[1, \infty),
$$


for some bounded and measurable function $\varepsilon$ satisfying $\lim _{u \rightarrow \infty} \varepsilon(u)=0$. Then $\psi$ is a (normalized) slowly varying function (see [1]). Furthermore, for $\alpha>0$ the function

$$
w_{\alpha, \psi}(x):=\left(1+|x|^{2}\right)^{\alpha / 2} \psi(|x|), \quad x \in \mathbb{R}^{d},
$$

belongs to $\mathcal{W}_{2}$. The function $\varphi(t)=\left(1+t^{2}\right)^{\alpha / 2} \psi(t)$ is associated with $w$ and a short calculation using Lemma 4.11 results in $\alpha_{\varphi}=\beta_{\varphi}=\alpha$. We give some examples for such weights:

(i) $w(x)=\left(1+|x|^{2}\right)^{\alpha / 2} \log \log \left(\mathrm{e}^{\mathrm{e}}+|x|^{2}\right)$;

(ii) $w(x)=\left(1+|x|^{2}\right)^{\alpha / 2} \log ^{\beta}\left(\mathrm{e}+|x|^{2}\right), \beta \in \mathbb{R}$;

(iii) $w(x)=\left(1+|x|^{2}\right)^{\alpha / 2}\left[\log \log \left(\mathrm{e}^{\mathrm{e}}+|x|^{2}\right)\right]^{\log \log \left(\mathrm{e}^{\mathrm{e}}+|x|^{2}\right)}$.

Further examples can be constructed by using the following facts.

(i) Any real power of a slowly varying function is again slowly varying.

(ii) If $\varphi$ is slowly varying, then $\varphi\left(t^{\alpha}\right), \alpha>0$, is slowly varying as well.

All these examples may be understood as weak perturbations (they do not change the indices) of the polynomial weights $w_{\alpha}$.

Weights with different indices

Here we present two examples.

Example 4.12. Let $a>1$ and let $0 \leqslant s_{0}<s_{1}<\infty$. Then we define

$$
\mathcal{X}(u):= \begin{cases}s_{0} & \text { if } \exp \left(a^{2 k}\right) \leqslant u<\exp \left(a^{2 k+1}\right), \\ s_{1} & \text { if } \exp \left(a^{2 k+1}\right) \leqslant u<\exp \left(a^{2 k+2}\right),\end{cases}
$$

$k \in \mathbb{N}_{0}$, and

$$
\varphi(t)=\exp \left\{\int_{1}^{t} \mathcal{X}(u) \frac{\mathrm{d} u}{u}\right\} .
$$

By Lemma 4.11 we have $\varphi \in \mathcal{V}$ and hence $\alpha_{\varphi}$ and $\beta_{\varphi}$ exist. Elementary but cumbersome calculations yield

$$
\alpha_{\varphi}=\frac{s_{0}+a s_{1}}{a+1} \quad \text { and } \quad \beta_{\varphi}=\frac{a s_{0}+s_{1}}{a+1} .
$$

The proof of (4.11) is based on Lemma 4.8 (iii).

First, observe that on the interval $\left[\exp \left(a^{2 k}\right), \exp \left(a^{2 k+1}\right)\right]$ we have the identity

$$
\frac{\varphi(t)}{t^{\gamma}}=c_{k} t^{s_{0}-\gamma} .
$$

Similarly,

$$
\frac{\varphi(t)}{t^{\gamma}}=C_{k} t^{s_{1}-\gamma}
$$


holds on $\left[\exp \left(a^{2 k+1}\right), \exp \left(a^{2 k+2}\right)\right]$. Consequently, the quantity $\varphi(t) / t^{\gamma}$ attains its maximal and minimal values in the corners of the appropriate intervals. Furthermore, we have

$$
\varphi\left(\exp \left(a^{2 L}\right)\right)=\exp \left(\left[s_{0}(a-1)+s_{1} a(a-1)\right] \frac{a^{2 L}-1}{a^{2}-1}\right)
$$

and

$$
\begin{aligned}
& \varphi\left(\exp \left(a^{2 L+1}\right)\right)=\exp \left(\left[s_{0}(a-1)+s_{1} a(a-1)\right] \frac{a^{2 L}-1}{a^{2}-1}+s_{0}\left(a^{2 L+1}-a^{2 L}\right)\right) \\
& L=1,2, \ldots
\end{aligned}
$$

We conclude that

$$
\begin{aligned}
\sup _{L=1,2, \ldots} \frac{\varphi\left(\exp \left(a^{2 L}\right)\right)}{\exp \left(\gamma a^{2 L}\right)}<\infty & \Longleftrightarrow \frac{s_{0}+a s_{1}}{a+1}-\gamma \leqslant 0 \\
\inf _{L=1,2, \ldots} \frac{\varphi\left(\exp \left(a^{2 L}\right)\right)}{\exp \left(\gamma a^{2 L}\right)}>0 & \Longleftrightarrow \frac{s_{0}+a s_{1}}{a+1}-\gamma \geqslant 0, \\
\sup _{L=1,2, \ldots} \frac{\varphi\left(\exp \left(a^{2 L+1}\right)\right)}{\exp \left(\gamma a^{2 L+1}\right)}<\infty & \Longleftrightarrow \frac{s_{0}+a s_{1}}{a(a+1)}+\frac{(a-1) s_{0}}{a}-\gamma \leqslant 0
\end{aligned}
$$

and

$$
\inf _{L=1,2, \ldots} \frac{\varphi\left(\exp \left(a^{2 L+1}\right)\right)}{\exp \left(\gamma a^{2 L+1}\right)}>0 \Longleftrightarrow \frac{s_{0}+a s_{1}}{a(a+1)}+\frac{(a-1) s_{0}}{a}-\gamma \geqslant 0 .
$$

Because

$$
\sup _{L \geqslant 1} \frac{\varphi\left(\exp \left(a^{2 L}\right)\right)}{\exp \left(\gamma a^{2 L}\right)}=\sup _{t \geqslant a^{2}} \frac{\varphi(t)}{t^{\gamma}}
$$

we derive from Lemma 4.8 (iii) that

$$
\alpha_{\varphi}=\max \left(\frac{s_{0}+a s_{1}}{a+1}, \frac{s_{0}+a s_{1}}{a(a+1)}+\frac{(a-1) s_{0}}{a}\right) .
$$

Similarly,

$$
\beta_{\varphi}=\min \left(\frac{s_{0}+a s_{1}}{a+1}, \frac{s_{0}+a s_{1}}{a(a+1)}+\frac{(a-1) s_{0}}{a}\right) .
$$

This proves the claim.

Example 4.13. Consider the following recursively defined sequence:

$$
j_{0}=0, \quad j_{1}=1, \quad j_{2 \ell}=2 j_{2 \ell-1}-j_{2 \ell-2} \quad \text { and } \quad j_{2 \ell+1}=2^{j_{2 \ell}}, \quad \ell \in \mathbb{N} .
$$

We define

$$
\xi(u):= \begin{cases}s_{0} & \text { if } 2^{j_{2 \ell}} \leqslant u<2^{j_{2 \ell+1}}, \\ s_{0}+s_{1} & \text { if } 2^{j_{2 \ell+1}} \leqslant u<2^{j_{2 \ell+2}}\end{cases}
$$


For $s_{0} \geqslant 0$ and $s_{1}>0$ the function

$$
\varphi(t):=\exp \left(\int_{1}^{t} \xi(u) \frac{\mathrm{d} u}{u}\right), \quad t \geqslant 1,
$$

belongs to $\mathcal{V}$ and is increasing. Arguing as in the first example one can show that

$$
\alpha_{\varphi}=s_{0}+\frac{1}{2} s_{1} \quad \text { and } \quad \beta_{\varphi}=s_{0} .
$$

For $s_{0}=0$ and $s_{1}=2$ this example has been treated in $[\mathbf{9}, \mathbf{2 7}]$.

\subsection{Entropy numbers of embeddings of weighted sequence spaces}

\subsubsection{The main result}

All our estimates will be based on the following inequalities.

For $0<p_{1} \leqslant p_{2} \leqslant \infty$ and all $n, N \in \mathbb{N}$ we have

$$
e_{n}\left(\mathrm{id}: \ell_{p_{1}}^{N} \rightarrow \ell_{p_{2}}^{N}\right) \sim \begin{cases}1 & \text { if } 1 \leqslant n \leqslant \log 2 N, \\ \left(\frac{\log (1+N / n)}{n}\right)^{1 / p_{1}-1 / p_{2}} & \text { if } \log 2 N \leqslant n \leqslant 2 N, \\ 2^{-n / 2 N} N^{1 / p_{2}-1 / p_{1}} & \text { if } 2 N \leqslant n .\end{cases}
$$

If $0<p_{2}<p_{1} \leqslant \infty$, then

$$
e_{n}\left(\mathrm{id}: \ell_{p_{1}}^{N} \rightarrow \ell_{p_{2}}^{N}\right) \sim 2^{-n / 2 N} N^{1 / p_{2}-1 / p_{1}} \quad \text { for all } n \in \mathbb{N} .
$$

If $1 \leqslant p_{1} \leqslant p_{2} \leqslant \infty$ this has been proved by Schütt [39]. For the remaining cases we refer to $[\mathbf{8}, 3.2 .2],[\mathbf{4 3}, 7.2,7.3]$ and $[\mathbf{2 2}]$.

Moreover, we specify the operator ideal. We choose $E=\ell_{r, \infty}$ as the Lorentz sequence space and write instead of $L_{E}^{(e)}(T)$ and $\mathcal{L}_{E}^{(e)}(X, Y)$ simply $L_{r, \infty}^{(e)}(T)$ and $\mathcal{L}_{r, \infty}^{(e)}(X, Y)$, respectively. Then we have

$$
L_{r, \infty}^{(e)}(T) \leqslant c \quad \text { if and only if } \sup _{n \in \mathbb{N}} n^{1 / r} e_{n}(T) \leqslant c .
$$

Our main result is the following theorem.

Theorem 4.14. Let $w \in \mathcal{W}_{2}$ and let $\varphi \in \mathcal{V}$ be an associated function in the sense of Definition 4.10.

(i) Suppose $d / p^{*}<\beta_{\varphi} \leqslant \alpha_{\varphi}<\delta$. Then

$$
e_{n}\left(\operatorname{id}: \ell_{q_{1}}\left(2^{j \delta}\left(\ell_{p_{1}}(w)\right)\right) \rightarrow \ell_{q_{2}}\left(\ell_{p_{2}}\right)\right) \sim n^{-\left(1 / p_{1}-1 / p_{2}\right)} \varphi\left(n^{1 / d}\right)^{-1} .
$$

(ii) Suppose that $d / p^{*}<\delta<\beta_{\varphi}$. Then

$$
e_{n}\left(\mathrm{id}: \ell_{q_{1}}\left(2^{j \delta}\left(\ell_{p_{1}}(w)\right)\right) \rightarrow \ell_{q_{2}}\left(\ell_{p_{2}}\right)\right) \sim n^{-\delta / d-\left(1 / p_{1}-1 / p_{2}\right)} .
$$




\section{Proof.}

Step 1. Preparation. We employ the same notation as in Substep 2.1 of the proof of Theorem 3.1. Thanks to Lemma 4.8 we have

$$
w\left(2^{-j} k\right) \sim \varphi\left(2^{-j}|k|\right) \sim \varphi\left(2^{i}\right) \quad \text { if }(j, k) \in I_{j, i}, \quad i \geqslant 0 .
$$

Monotonicity arguments and elementary properties of the entropy numbers yield

$$
\begin{aligned}
e_{n}\left(P_{j, i}: B_{1} \rightarrow B_{2}\right) & \leqslant \frac{1}{\inf _{k \in I_{j, i}} w\left(2^{-j} k\right)} 2^{-j \delta} e_{n}\left(\mathrm{id}: \ell_{p_{1}}^{M_{j, i}} \rightarrow \ell_{p_{2}}^{M_{j, i}}\right) \\
& \leqslant c \frac{1}{2^{j \delta} \varphi\left(2^{i}\right)} e_{n}\left(\mathrm{id}: \ell_{p_{1}}^{M_{j, i}} \rightarrow \ell_{p_{2}}^{M_{j, i}}\right)
\end{aligned}
$$

with a constant $c$ independent of $n, j$ and $i$.

Step 2. Now the operator ideal comes into play. Using (4.14) and (4.18) we find

$$
L_{r, \infty}^{(e)}\left(P_{j, i}\right) \leqslant c \frac{1}{2^{j \delta} \varphi\left(2^{i}\right)} L_{r, \infty}^{(e)}\left(\mathrm{id}: \ell_{p_{1}}^{M_{j, i}} \rightarrow \ell_{p_{2}}^{M_{j, i}}\right) .
$$

To shorten notation define $1 / p:=1 / p_{1}-1 / p_{2}$. The known asymptotic behaviour of the entropy numbers $e_{n}\left(\mathrm{id}: \ell_{p_{1}}^{N} \rightarrow \ell_{p_{2}}^{N}\right.$ ) (see (4.12) and (4.13)) implies that

$$
L_{r, \infty}^{(e)}\left(\mathrm{id}: \ell_{p_{1}}^{N} \rightarrow \ell_{p_{2}}^{N}\right) \sim N^{1 / r-1 / p} \text { if } \frac{1}{r}>\max \left(0, \frac{1}{p}\right) .
$$

Under the assumption $1 / r>\max (0,1 / p)$ we conclude from (3.7) and (4.20) that

$$
L_{r, \infty}^{(e)}\left(\mathrm{id}: \ell_{p_{1}}^{M_{j, i}} \rightarrow \ell_{p_{2}}^{M_{j, i}}\right) \sim 2^{d(j+i)(1 / r-1 / p)}
$$

and consequently that

$$
L_{r, \infty}^{(e)}\left(P_{j, i}\right) \leqslant c \frac{1}{2^{j \delta} \varphi\left(2^{i}\right)} 2^{d(j+i)(1 / r-1 / p)}
$$

Now, for given $M \in \mathbb{N}_{0}$, let

$$
P:=\sum_{m=0}^{M} \sum_{j+i=m} P_{j, i} \quad \text { and } \quad Q:=\sum_{m=M+1}^{\infty} \sum_{j+i=m} P_{j, i} .
$$

Substep 2.1. Estimate of $L_{r, \infty}^{(e)}(P)$. Recall that for any $r>0$ there exists an equivalent $\varrho$-norm on $\mathcal{L}_{r, \infty}^{(e)}$, with some $0<\varrho \leqslant 1$. Hence, Theorem 4.3, (4.22) and Lemma 4.9 yield

$$
\begin{aligned}
L_{r, \infty}^{(e)}(P)^{\varrho} & \leqslant \sum_{m=1}^{M} \sum_{j+i=m} L_{r, \infty}^{(e)}\left(P_{j, i}\right)^{\varrho} \\
& \leqslant c_{1} \sum_{m=1}^{M} \sum_{j+i=m} \frac{1}{2^{j \varrho \delta} \varphi^{\varrho}\left(2^{i}\right)} 2^{\varrho d m(1 / r-1 / p)}
\end{aligned}
$$




$$
\begin{aligned}
& \leqslant c_{2} \sum_{m=1}^{M} 2^{\varrho d m(1 / r-1 / p)} \sum_{j=0}^{m} \frac{1}{2^{j \varrho \delta} \varphi^{\varrho}\left(2^{m-j}\right)} \\
& \leqslant c_{3} \sum_{m=1}^{M} 2^{\varrho d m(1 / r-1 / p)} \times \begin{cases}1 / \varphi^{\varrho}\left(2^{m}\right) & \text { if } \alpha_{\varphi}<\delta, \\
2^{-m \varrho \delta} & \text { if } \beta_{\varphi}>\delta .\end{cases}
\end{aligned}
$$

Let us first consider the case $\alpha_{\varphi}<\delta$. If $r$ is chosen such that

$$
\frac{1}{r}>\max \left(0, \frac{1}{p}\right) \text { and } d\left(\frac{1}{r}-\frac{1}{p}\right)-\alpha_{\varphi}>0
$$

then

$$
L_{r, \infty}^{(e)}(P)^{\varrho} \leqslant c_{3} 2^{\varrho d M(1 / r-1 / p)} \sum_{m=1}^{M} \frac{2^{-\varrho d(M-m)(1 / r-1 / p)}}{\varphi^{\varrho}\left(2^{m}\right)}
$$

and (4.5) gives

$$
L_{r, \infty}^{(e)}(P) \leqslant c_{3} \frac{2^{d M(1 / r-1 / p)}}{\varphi\left(2^{M}\right)} .
$$

In view of (4.14) this implies that

$$
e_{2^{M d}}\left(P: B_{1} \rightarrow B_{2}\right) \leqslant c_{3} 2^{d M\left(-1 / p_{1}+1 / p_{2}\right)}\left(\varphi\left(2^{M}\right)\right)^{-1} .
$$

Now we consider the case $\beta_{\varphi}>\delta$. Then, in a similar manner as above, we find that

$$
e_{2^{M d}}\left(P: B_{1} \rightarrow B_{2}\right) \leqslant c 2^{d M\left(-1 / p_{1}+1 / p_{2}-\delta / d\right)}
$$

if

$$
\frac{1}{r}>\max \left(0, \frac{1}{p}\right) \quad \text { and } \quad d\left(\frac{1}{r}-\frac{1}{p}\right)-\delta>0 .
$$

Observe that (4.24) and (4.27) are satisfied if $r$ is chosen small enough.

Substep 2.2. Estimate of $L_{r, \infty}^{(e)}(Q)$. We proceed as in Substep 2.1 and obtain

$$
L_{r, \infty}^{(e)}(Q)^{\varrho} \leqslant c_{1} \sum_{m=M+1}^{\infty} 2^{\varrho d m(1 / r-1 / p)} \times \begin{cases}\left(\varphi\left(2^{m}\right)\right)^{-\varrho} & \text { if } \alpha_{\varphi}<\delta \\ 2^{-m \varrho \delta} & \text { if } \beta_{\varphi}>\delta .\end{cases}
$$

When $\alpha_{\varphi}<\delta$ this leads, via (4.7), to

$$
L_{r, \infty}^{(e)}(Q) \leqslant c_{2} 2^{d M(1 / r-1 / p)}\left(\varphi\left(2^{M}\right)\right)^{-1}
$$

if

$$
\frac{1}{r}-\frac{1}{p}<\frac{\beta_{\varphi}}{d}
$$

Because $\beta_{\varphi}>d / p^{*}=d \max (0,-1 / p)$, we have

$$
\max \left(0, \frac{1}{p}\right)<\frac{\beta_{\varphi}}{d}+\frac{1}{p}
$$


Hence, there exists an appropriate $r$ with

$$
\max \left(0, \frac{1}{p}\right)<\frac{1}{r}<\frac{\beta_{\varphi}}{d}+\frac{1}{p} .
$$

This gives

$$
e_{2^{M d}}\left(Q: B_{1} \rightarrow B_{2}\right) \leqslant c_{3} 2^{d M\left(-1 / p_{1}+1 / p_{2}\right)}\left(\varphi\left(2^{M}\right)\right)^{-1} .
$$

If $\beta_{\varphi}>\delta$, using the same types of argument, we derive

$$
e_{2^{M d}}\left(Q: B_{1} \rightarrow B_{2}\right) \leqslant c 2^{d M\left(-1 / p_{1}+1 / p_{2}-\delta / d\right)},
$$

where the number $r$ has to be chosen such that

$$
\max \left(0, \frac{1}{p}\right)<\frac{1}{r}<\frac{\delta}{d}+\frac{1}{p}
$$

(which is possible because $\delta>d / p^{*}$ ). In summary, we get

$$
\begin{aligned}
e_{2^{M d+1}}\left(\text { id }: B_{1} \rightarrow B_{2}\right) & \leqslant e_{2^{M d}}\left(P: B_{1} \rightarrow B_{2}\right)+e_{2^{M d}}\left(Q: B_{1} \rightarrow B_{2}\right) \\
& \leqslant c 2^{d M\left(-1 / p_{1}+1 / p_{2}\right)} \times \begin{cases}\left(\varphi\left(2^{M}\right)\right)^{-1} & \text { if } \alpha_{\varphi}<\delta, \\
2^{-M \delta} & \text { if } \beta_{\varphi}>\delta,\end{cases}
\end{aligned}
$$

and by monotonicity of the entropy numbers the upper estimates in (4.16) and (4.15) follow.

Step 3. For the estimate from below we consider the following commutative diagram:

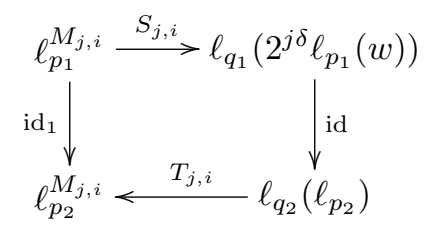

Here

$$
\begin{aligned}
\left(T_{j, i} \lambda\right)_{\phi(u, v)} & :=\lambda_{u, v}, \quad(u, v) \in I_{j, i}, \\
\left(S_{j, i} \eta\right)_{u, v} & := \begin{cases}\eta_{\varphi(u, v)} & \text { if }(u, v) \in I_{j, i} \\
0 & \text { otherwise }\end{cases}
\end{aligned}
$$

and $\phi$ denotes a bijection of $I_{j, i}$ onto $\left\{1,2, \ldots, M_{j, i}\right\}$. Observe that

$$
\left\|T_{j, i}: \ell_{q_{2}}\left(\ell_{p_{2}}\right) \rightarrow \ell_{p_{2}}^{M_{j, i}}\right\|=1
$$

and

$$
\left\|S_{j, i}: \ell_{p_{1}}^{M_{j, i}} \rightarrow \ell_{q_{1}}\left(2^{j \delta} \ell_{p_{1}}(w)\right)\right\| \sim 2^{j \delta} \varphi\left(2^{i}\right)
$$


Hence we obtain

$$
\begin{aligned}
e_{n}\left(\mathrm{id}_{1}: \ell_{p_{1}}^{M_{j, i}} \rightarrow \ell_{p_{2}}^{M_{j, i}}\right) & \leqslant\left\|S_{j, i}\right\|\left\|T_{j, i}\right\| e_{n}\left(\mathrm{id}: B_{1} \rightarrow B_{2}\right) \\
& \leqslant c 2^{j \delta} \varphi\left(2^{i}\right) e_{n}\left(\mathrm{id}: B_{1} \rightarrow B_{2}\right) .
\end{aligned}
$$

Using once more the characterization from (4.12) and (4.13) we find with $n=2^{(j+i) d} \sim$ $M_{j, i}$ that

$$
n^{-(\delta / d)-\left(1 / p_{1}\right)+\left(1 / p_{2}\right)} \leqslant c e_{n}\left(\text { id }: B_{1} \rightarrow B_{2}\right) \quad \text { if } i=0
$$

and

$$
n^{-\left(1 / p_{1}\right)+\left(1 / p_{2}\right)}\left(\varphi\left(n^{1 / d}\right)\right)^{-1} \leqslant c e_{n}\left(\text { id }: B_{1} \rightarrow B_{2}\right) \quad \text { if } j=0 .
$$

The estimate for the remaining $n$ follows by monotonicity of the entropy numbers and the properties of $\varphi$. This finishes the proof.

\subsubsection{Examples}

In this subsection we consider polynomial weights $w_{\alpha}(x)=\left(1+|x|^{2}\right)^{\alpha / 2}$ and polynomial weights with small perturbations $w_{\alpha, \psi}(x)=\left(1+|x|^{2}\right)^{\alpha / 2} \psi(x)$. In these situations we always have $\alpha_{\varphi}=\beta_{\varphi}=\alpha$.

\section{Polynomial weights}

To begin with, we reformulate Theorem 3.1 in this situation.

Corollary 4.15. Let $w_{\alpha}$ be as in (4.9) for some $\alpha>0$. Then the embedding

$$
\ell_{q_{1}}\left(2^{j \delta} \ell_{p_{1}}\left(w_{\alpha}\right)\right) \hookrightarrow \ell_{q_{2}}\left(\ell_{p_{2}}\right)
$$

is compact if and only if $\min (\delta, \alpha)>d / p^{*}$.

Applying Theorem 4.14 in these particular cases yields the following.

Corollary 4.16. Let $\min (\alpha, \delta)>d / p^{*}$. Suppose $\delta \neq \alpha$. Then

$$
e_{n}\left(\operatorname{id}: \ell_{q_{1}}\left(2^{j \delta} \ell_{p_{1}}\left(w_{\alpha}\right)\right) \rightarrow \ell_{q_{2}}\left(\ell_{p_{2}}\right)\right) \sim n^{-\min (\alpha, \delta) / d-1 / p_{1}+1 / p_{2}}
$$

holds for all $n \in \mathbb{N}$.

Remark 4.17. Note that the result does not depend on the microscopic parameters $q_{1}$ and $q_{2}$. This is different in the case in which $\alpha=\delta$ (see [25]).

\section{Small perturbations of polynomial weights}

This time Theorem 3.1 reads as follows.

Corollary 4.18. Let $w_{\alpha, \psi}$ be as in (4.10) for some $\alpha>0$. The embedding

$$
\ell_{q_{1}}\left(2^{j \delta} \ell_{p_{1}}\left(w_{\alpha, \psi}\right)\right) \hookrightarrow \ell_{q_{2}}\left(\ell_{p_{2}}\right)
$$


is compact if and only if one of the following conditions is satisfied:

(i) $\min (\delta, \alpha)>d / p^{*}$;

(ii) $\delta>\alpha=d / p^{*}$ and $\int_{1}^{\infty} \psi(t)^{-p^{*}}(\mathrm{~d} t / t)<\infty$.

Theorem 4.14 implies the following corollary.

\section{Corollary 4.19 .}

(i) Suppose that $d / p^{*}<\alpha<\delta$. Then

$$
e_{n}\left(\mathrm{id}: \ell_{q_{1}}\left(2^{j \delta} \ell_{p_{1}}\left(w_{\alpha, \psi}\right)\right) \rightarrow \ell_{q_{2}}\left(\ell_{p_{2}}\right)\right) \sim n^{-\alpha / d-\left(1 / p_{1}-1 / p_{2}\right)} \psi\left(n^{1 / d}\right)^{-1}
$$

holds for all $n \in \mathbb{N}$.

(ii) Suppose that $d / p^{*}<\delta<\alpha$. Then

$$
e_{n}\left(\operatorname{id}: \ell_{q_{1}}\left(2^{j \delta} \ell_{p_{1}}\left(w_{\alpha, \psi}\right)\right) \rightarrow \ell_{q_{2}}\left(\ell_{p_{2}}\right)\right) \sim n^{-\delta / d-\left(1 / p_{1}-1 / p_{2}\right)}
$$

holds for all $n \in \mathbb{N}$.

\section{Entropy numbers of embeddings of weighted function spaces}

Based on Proposition 2.8 we transfer the results of $\S 4$ for weighted sequence spaces step by step to weighted function spaces. For simplicity we concentrate on the situation where the target space is an unweighted function space. This restriction can be removed immediately by using (4.2).

\subsection{The non-limiting case for polynomial weights}

Let $w_{\alpha}$ be as in (4.9) and let $\delta$ be given by (4.1). Then the counterpart of Corollary 4.15 reads as follows.

Corollary 5.1. The embedding $B_{p_{1}, q_{1}}^{s_{1}}\left(\mathbb{R}^{d}, w_{\alpha}\right) \hookrightarrow B_{p_{2}, q_{2}}^{s_{2}}\left(\mathbb{R}^{d}\right)$ is compact if and only if $\min (\delta, \alpha)>d / p^{*}$.

Remark 5.2. Of course, the if part of the assertion in Corollary 5.1 is known (see, for example, $[\mathbf{8}, 4.2 .3])$. The only if part is also partially known.

The counterpart of Corollary 4.16 is the following theorem.

Theorem 5.3.

(i) Let $d / p^{*}<\delta<\alpha$. Then

$$
e_{n}\left(\mathrm{id}: B_{p_{1}, q_{1}}^{s_{1}}\left(\mathbb{R}^{d}, w_{\alpha}\right) \rightarrow B_{p_{2}, q_{2}}^{s_{2}}\left(\mathbb{R}^{d}\right)\right) \sim n^{-\left(s_{1}-s_{2}\right) / d} .
$$

(ii) Let $d / p^{*}<\alpha<\delta$. Then

$$
e_{n}\left(\operatorname{id}: B_{p_{1}, q_{1}}^{s_{1}}\left(\mathbb{R}^{d}, w_{\alpha}\right) \rightarrow B_{p_{2}, q_{2}}^{s_{2}}\left(\mathbb{R}^{d}\right)\right) \sim n^{-\alpha / d+1 / p_{2}-1 / p_{1}} .
$$


Remark 5.4. A rough interpretation is as follows. If the weight is increasing fast enough near infinity, i.e. if $\alpha>\delta$, then the asymptotic entropy behaviour of the corresponding embeddings is the same as for embeddings of unweighted Besov spaces defined on a compact domain with smooth boundary (see $[\mathbf{8}, 3.3]$ ). If the weight is not increasing fast enough, i.e. if $\alpha<\delta$, then the weight has a direct influence on the asymptotic behaviour of the entropy numbers.

Remark 5.5. The estimates in (i) and the estimate from below in (ii) are covered by [15] (see also $[\mathbf{8}, 4.2 .3]$ ). Also, in the case when $p_{2} \leqslant p_{1}$ a proof of the estimate from above is given in $[\mathbf{8}, 4.3 .2]$ (see also [16]). The correctness of (ii) for all cases was conjectured in [14]. A different proof of (ii) was given recently in [44] and in [17].

\subsection{Small perturbations of polynomial weights: the non-limiting case}

Let $w_{\alpha, \psi}$ be as in (4.10) for some $\alpha>0$. By Lemma 4.11 we know that $w_{\alpha, \psi} \in \mathcal{W}_{2}$. We have to check under which conditions such a weight also belongs to the class $\mathcal{W}_{1}$.

Lemma 5.6. Suppose that the function $\xi$ is infinitely differentiable and bounded together with all its derivatives on $(1, \infty)$. Let $\varphi(t)=\exp \left(\int_{1}^{t} \xi(u) \mathrm{d} u / u\right), t \geqslant 1$. Let $\psi: \mathbb{R} \rightarrow \mathbb{R}$ be a positive $C^{\infty}$-function such that $\psi(t)=\varphi(t), t \geqslant 1$, and $\psi(t)=1$ if $|t| \leqslant \frac{1}{2}$. Then the function $w(x)=\psi(|x|)$ belongs to $\mathcal{W}_{1}$.

Proof. On $(1, \infty)$ we have the identity

$$
\xi(t)=t \frac{\varphi^{\prime}(t)}{\varphi(t)} .
$$

Since $\xi$ is bounded on $[1, \infty)$ we conclude that $\varphi^{\prime}(t) / \varphi(t)$ is bounded on this interval. Rearranging the above identity we see that

$$
\psi^{(m+1)}(t)=\left(\frac{\xi(t)}{t} \psi(t)\right)^{(m)} .
$$

All derivatives of the quotient $\xi(t) / t$ remain bounded on $[1, \infty)$. Hence we have

$$
\left|\psi^{(m+1)}(t)\right| \leqslant c_{m} \sum_{j=0}^{m}\left|\psi^{(j)}(t)\right|
$$

for some constant $c_{m}$. Now an induction argument yields the conclusion.

Remark 5.7. It is an easy exercise to check that the following functions belong to $\mathcal{W}_{1} \cap \mathcal{W}_{2}$

(i) $w(x)=\left(1+|x|^{2}\right)^{\alpha / 2} \log \log \left(\mathrm{e}^{\mathrm{e}}+|x|^{2}\right)$.

(ii) $w(x)=\left(1+|x|^{2}\right)^{\alpha / 2} \log ^{\beta}\left(\mathrm{e}+|x|^{2}\right), \beta \in \mathbb{R}$.

(iii) $w(x)=\left(1+|x|^{2}\right)^{\alpha / 2}\left[\log \log \left(\mathrm{e}^{\mathrm{e}}+|x|^{2}\right)\right]^{\log \log \left(\mathrm{e}^{\mathrm{e}}+|x|^{2}\right)}$. 
Then the counterpart of Corollary 4.18 reads as follows.

Corollary 5.8. Suppose that $w_{\alpha, \psi} \in \mathcal{W}_{1} \cap \mathcal{W}_{2}$. Then the embedding

$$
B_{p_{1}, q_{1}}^{s_{1}}\left(\mathbb{R}^{d}, w_{\alpha, \psi}\right) \hookrightarrow B_{p_{2}, q_{2}}^{s_{2}}\left(\mathbb{R}^{d}\right)
$$

is compact if and only if either $\min (\delta, \alpha)>d / p^{*}$ or $\delta>\alpha=d / p^{*}>0$ and

$$
\int_{1}^{\infty} \psi(t)^{-p^{*}} \frac{\mathrm{d} t}{t}<\infty
$$

Furthermore, Corollary 4.19 yields the following theorem.

Theorem 5.9. Suppose that $d / p^{*}<\alpha<\delta$. Then

$$
e_{n}\left(\mathrm{id}: B_{p_{1}, q_{1}}^{s_{1}}\left(\mathbb{R}^{d}, w_{\alpha, \psi}\right) \rightarrow B_{p_{2}, q_{2}}^{s_{2}}\left(\mathbb{R}^{d}\right)\right) \sim n^{-\alpha / d-\left(1 / p_{1}-1 / p_{2}\right)} \psi\left(n^{1 / d}\right)^{-1} .
$$

Remark 5.10. In the case where $\alpha>\delta>d / p^{*}$ the behaviour of the entropy numbers is also well known. We refer to Remark 5.17 for further details.

Remark 5.11. In $[\mathbf{1 3}, 14]$ only the case $\psi(x)=\log ^{\beta}(\mathrm{e}+|x|), \beta \in \mathbb{R}$, is treated. In addition we improved the author's estimate from above in the case where $p_{1}<p_{2}$.

\subsection{The general case}

Finally, we formulate our result in the general situation.

Corollary 5.12. Let $w \in \mathcal{W}_{1} \cap \mathcal{W}_{2}$, and let $\varphi \in \mathcal{V}$ be an associated function in the sense of Definition 4.10.

(i) Let $p^{*}=\infty$. Then the embedding $B_{p_{1}, q_{1}}^{s_{1}}\left(\mathbb{R}^{d}, w\right) \hookrightarrow B_{p_{2}, q_{2}}^{s_{2}}\left(\mathbb{R}^{d}\right)$ is compact if and only if $\delta>0$ and $\lim _{t \rightarrow \infty} \varphi(t)=\infty$.

(ii) Let $0<p^{*}<\infty$. Then the embedding $B_{p_{1}, q_{1}}^{s_{1}}\left(\mathbb{R}^{d}, w\right) \hookrightarrow B_{p_{2}, q_{2}}^{s_{2}}\left(\mathbb{R}^{d}\right)$ is compact if and only if

$$
\delta>d / p^{*} \quad \text { and } \quad \int_{1}^{\infty} \varphi(t)^{-p^{*}} s^{d} \frac{\mathrm{d} s}{s} .
$$

Furthermore, Theorem 4.14 yields the following theorem.

Theorem 5.13. Let $w \in \mathcal{W}_{1} \cap \mathcal{W}_{2}$, and let $\varphi \in \mathcal{V}$ be an associated function in the sense of Definition 4.10.

(i) Suppose that $d / p^{*}<\beta_{\varphi} \leqslant \alpha_{\varphi}<\delta$. Then

$$
e_{n}\left(\mathrm{id}: B_{p_{1}, q_{1}}^{s_{1}}\left(\mathbb{R}^{d}, w\right) \rightarrow B_{p_{2}, q_{2}}^{s_{2}}\left(\mathbb{R}^{d}\right)\right) \sim n^{-\left(1 / p_{1}-1 / p_{2}\right)} \varphi\left(n^{1 / d}\right)^{-1} .
$$

(ii) Suppose that $d / p^{*}<\delta<\beta_{\varphi}$. Then

$$
e_{n}\left(\operatorname{id}: B_{p_{1}, q_{1}}^{s_{1}}\left(\mathbb{R}^{d}, w\right) \rightarrow B_{p_{2}, q_{2}}^{s_{2}}\left(\mathbb{R}^{d}\right)\right) \sim n^{-\delta / d-\left(1 / p_{1}-1 / p_{2}\right)} .
$$




\section{A special example}

We consider the weight function with different indices introduced in Example 4.12. Let $a, s_{0}, s_{1}, \mathcal{X}$ and $\varphi$ be defined as there. Since the function $\mathcal{X}$ is not smooth, we have to replace this function by a smooth version of it. To this end, let $\varrho \in C_{0}^{\infty}(\mathbb{R})$ be a function such that $\varrho \geqslant 0, \operatorname{supp} \varrho \subset[-1,0]$ and $\int \varrho(u) \mathrm{d} u=1$. We define the function $\xi$ by

$$
\xi(u):=\int \mathcal{X}(y) \varrho(u-y) \mathrm{d} y .
$$

Let $\psi: \mathbb{R} \rightarrow \mathbb{R}$ be an even positive $C^{\infty}$-function such that $\psi(t)=\exp \left(\int_{1}^{t} \xi(u) \mathrm{d} u / u\right)$, $t \geqslant 1$, and $\psi(t)=1$ if $|t| \leqslant \frac{1}{2}$. Then, applying Lemma 5.6, we conclude that the function $w(x)=\psi(|x|)$ belongs to $\mathcal{W}_{1}$. Moreover, elementary calculations show that (4.11) implies

$$
\alpha_{\psi}=\frac{s_{0}+a s_{1}}{a+1} \quad \text { and } \quad \beta_{\psi}=\frac{a s_{0}+s_{1}}{a+1} .
$$

So we find the following corollary in this particular situation (see Theorem 4.14).

\section{Corollary 5.14.}

(i) Suppose that

$$
\frac{d}{p^{*}}<\frac{a s_{0}+s_{1}}{a+1} \leqslant \frac{s_{0}+a s_{1}}{a+1}<\delta
$$

Then

$$
e_{n}\left(\mathrm{id}: B_{p_{1}, q_{1}}^{s_{1}}\left(\mathbb{R}^{d}, w\right) \rightarrow B_{p_{2}, q_{2}}^{s_{2}}\left(\mathbb{R}^{d}\right)\right) \sim n^{-\left(1 / p_{1}-1 / p_{2}\right)} \psi\left(n^{1 / d}\right)^{-1} .
$$

(ii) Suppose that

$$
\frac{d}{p^{*}}<\delta<\frac{a s_{0}+s_{1}}{a+1}
$$

Then

$$
e_{n}\left(\mathrm{id}: B_{p_{1}, q_{1}}^{s_{1}}\left(\mathbb{R}^{d}, w\right) \rightarrow B_{p_{2}, q_{2}}^{s_{2}}\left(\mathbb{R}^{d}\right)\right) \sim n^{-\delta / d-\left(1 / p_{1}-1 / p_{2}\right)} .
$$

Remark 5.15. Note that in part (i) of the preceding corollary the behaviour of the entropy numbers reflects the behaviour of the weight, as in the polynomial case.

Remark 5.16. Since our results do not depend on the microscopic parameters $q_{1}$ and $q_{2}$, all of them extend to Lizorkin-Triebel spaces $F_{p, q}^{s}\left(\mathbb{R}^{d}, w\right)$. This scale generalizes weighted Sobolev spaces $W_{p}^{s}\left(\mathbb{R}^{d}, w\right)$. Indeed, if $1<p<\infty$ then the following holds: $F_{p, 2}^{s}\left(\mathbb{R}^{d}, w\right)=W_{p}^{s}\left(\mathbb{R}^{d}, w\right)$.

Remark 5.17. Finally, let us mention some open questions.

(i) Let $w$ be a weight with $\lim _{|x| \rightarrow \infty} w(x)=\infty$ and such that the indices of the associated function $\varphi$ satisfy $\alpha_{\varphi}=\beta_{\varphi}=0$. What is the exact asymptotic entropy behaviour of the embeddings of the corresponding weighted Besov spaces? In the forthcoming paper $[\mathbf{2 4}]$ this question is solved for logarithmic weights, and in this situation some new phenomena occur. 
(ii) Interpreting Theorem 5.13 (ii) we see, if the weight grows fast enough near infinity, that the entropy numbers of the embedding of the weighted spaces behave like the entropy numbers of the embedding of the unweighted spaces defined on the unit ball. It would be of interest to characterize the class of all weights $w$ such that

$$
e_{n}\left(\operatorname{id}: B_{p_{1}, q_{1}}^{s_{1}}\left(\mathbb{R}^{d}, w\right) \rightarrow B_{p_{2}, q_{2}}^{s_{2}}\left(\mathbb{R}^{d}\right)\right) \sim n^{-\delta / d-\left(1 / p_{1}-1 / p_{2}\right)} .
$$

First results in this direction have been obtained in $[\mathbf{1 4}, \mathbf{2 3}]$.

(iii) Connected with the second problem is also the following question. Let $w_{\alpha, \psi}$ be a small perturbation of a polynomial weight and consider the limiting case $\alpha=\delta$. How do the entropy numbers behave in this situation? For first results we again refer to $[\mathbf{1 4}, \mathbf{2 3}]$.

Acknowledgements. T.K. was supported in part by Ministerio de Educación y Ciencia de España (project no. MTM2004-01888).

\section{References}

1. N. H. Bingham, C. M. Goldie And J. L. Teugels, Regular variation (Cambridge University Press, 1987).

2. G. Bourdaud, Ondelettes et espaces de Besov, Rev. Mat. Iber. 11 (1995), 477-512.

3. M. BRICCHI AND S. MourA, Complements on growth envelopes of spaces with generalised smoothness in the sub-critical case, Z. Analysis Anwend. 22 (2003), 383-398.

4. H.-Q. BuY, Weighted Besov and Triebel spaces: interpolation by the real method, Hiroshima Math. J. 12 (1982), 581-605.

5. H.-Q. Buy, M. Paluszyński and M. H. TAibleson, A maximal function characterization of weighted Besov-Lipschitz and Triebel-Lizorkin spaces, Studia Math. 119 (1996), 219-246.

6. B. CARl AND I. Stephani, Entropy, compactness and the approximation of operators (Cambridge University Press, 1990).

7. F. Cobos And D. L. Fernandez, Hardy-Sobolev spaces and Besov spaces with a function parameter, Springer Lecture Notes in Mathematics, Volume 1302, pp. 158-170 (Springer, 1986).

8. D. E. Edmunds And H. TRIEBel, Function spaces, entropy numbers, differential operators (Cambridge University Press, 1996).

9. W. FARKAS AND H.-G. LEOPOLD, Characterisation of function spaces of generalised smoothness, Annli Mat. Pura Appl. 185 (2006), 1-62.

10. J. Franke, Fourier-Multiplikatoren, Littlewood-Paley-Theoreme und Approximation durch ganze analytische Funktionen in gewichteten Funktionenräumen, Forschungsergebnisse FSU Jena, N/86/8 (1986).

11. J. Garcia-Cuerva and K. S. Kazarian, Spline wavelet bases of weighted $L_{p}$ spaces, $1 \leqslant p<\infty$, Proc. Am. Math. Soc. 123 (1995), 433-439.

12. J. GUSTAVSSON, A function parameter in connection with interpolation of Banach spaces, Math. Scand. 42 (1978), 289-305.

13. D. Haroske, Some limiting embeddings in weighted function spaces and related entropy numbers, Forschungsergebnisse FSU Jena, Math/Inf/97/04 (1997).

14. D. Haroske, Embeddings of some weighted function spaces on $\mathbb{R}^{n}$; entropy and approximation numbers, A survey of some recent results, Analele Univ. Craiova Mat.-Inform. 24 (1997), 1-44. 
15. D. HAROSKE AND H. TRIEBEL, Entropy numbers in weighted function spaces and eigenvalue distributions of some degenerate pseudodifferential operators, I, Math. Nachr. 167 (1994), 131-156.

16. D. HAROSKE AND H. TRIEBEL, Entropy numbers in weighted function spaces and eigenvalue distributions of some degenerate pseudodifferential operators, II, Math. Nachr. 168 (1994), 109-137.

17. D. HAROSKe AND H. TRIEBEL, Wavelet bases and entropy numbers in weighted function spaces, Math. Nachr. 278 (2005), 108-132.

18. E. Hille AND R. S. Phillips, Functional analysis and semi-groups (American Mathematical Society, Providence, RI, 1957).

19. J.-P. Kahane And P.-G. LemariÉ-Rieusset, Fourier series and wavelets (Gordon and Breach, New York, 1995).

20. T. F. KALugina, Interpolation of Banach spaces with a functional parameter, The reiteration theorem, Vestn. Moskov. Univ. Ser. I 30 (1975), 68-77.

21. H. KÖNIG, Eigenvalue distribution of compact operators (Birkhäuser, Basel, 1986).

22. T. KüHn, A lower estimate for entropy numbers, J. Approx. Theory 110 (2001), 120-124.

23. T. KüHN, H.-G. LEOPOLD, W. Sickel AND L. SkrzypczAK, Entropy numbers of embeddings of weighted Besov spaces II, Jenaer Schriften zur Mathematik und Informatik, Math/Inf/13/03 (2003).

24. T. KüHn, H.-G. LeOpold, W. Sickel And L. SkrzypczaK, Entropy numbers of embeddings of weighted Besov spaces, III, Weights of logarithmic type, preprint (2005).

25. T. Kühn, H.-G. Leopold, W. Sickel And L. Skrzypczak, Entropy numbers of embeddings of weighted Besov spaces, I, Constr. Approx. 23 (2006), 61-77.

26. P.-G. Lemarié-Rieusset, Ondelettes et poids de Muckenhoupt, Studia Math. 108 (1994), 127-147.

27. H.-G. LEOPOLD, Limiting embeddings for sequence spaces and entropy numbers, Forschungsergebnisse FSU Jena, Math/Inf/98/27 (1998).

28. H.-G. LEOPOLD, Embeddings and entropy numbers for general weighted sequence spaces, The non-limiting case, Georgian Math. J. 7 (2000), 1-13.

29. J. LÖFStröm, Interpolation of weighted spaces of differentiable functions on $\mathbb{R}^{d}$, Annli Mat. Pura Appl. 132 (1982), 189-214.

30. Y. MEYER, Wavelets and operators (Cambridge University Press, 1992).

31. J. Peetre, New thoughts on Besov spaces (Duke University Press, Durham, NC, 1976).

32. A. PIEtsch, Operator ideals (VEB, Berlin, 1978).

33. A. Pietsch, Eigenvalues and s-numbers (Akademische Verlagsgesellschaft Geest \& Portig, Leipzig, 1987).

34. S. Roudenko, Matrix-valued Besov spaces, Trans. Am. Math. Soc. 355 (2002), 273-314.

35. V. S. Rychkov, Littlewood-Paley theory and function spaces with $A_{p}^{\text {loc }}$ weights, Math. Nachr. 224 (2001), 145-180.

36. H.-J. SChMEISSER AND H. TRIEBEL, Topics in Fourier analysis and function spaces (Akademische Verlagsgesellschaft Geest \& Portig, Leipzig, 1987).

37. T. Schотт, Function spaces with exponential weights, I, Math. Nachr. 189 (1998), 221242 .

38. T. Sснотт, Function spaces with exponential weights, II, Math. Nachr. 196 (1998), 231250.

39. C. Sснӥтт, Entropy numbers of diagonal operators between symmetric Banach spaces, J. Approx. Theory 40 (1984), 121-128.

40. H. TRIEBEL, Interpolation theory, function spaces, differential operators (North-Holland, Amsterdam, 1978).

41. H. TRIEBEL, Theory of function spaces (Birkhäuser, Basel, 1983).

42. H. TRIEBeL, Theory of function spaces, II (Birkhäuser, Basel, 1992). 
43. H. TRIEBEL, Fractals and spectra (Birkhäuser, Basel, 1998).

44. H. TRIEBEL, Weighted and radial function spaces: decompositions and entropy numbers, handwritten notes, Jena (2001).

45. P. Wojtaszczyk, A mathematical introduction to wavelets (Cambridge University Press, 1997). 